\title{
Helicoidal particles and swimmers in a flow at low Reynolds number
}

\author{
Kenta Ishimoto $\dagger$ \\ Research Institute for Mathematical Sciences, Kyoto University, Kyoto, 606-8502, Japan
}

(Received 7 October 2019; revised 16 January 2020; accepted 15 February 2020)

In this paper, we consider the dynamics of a helicoidal object, which can be either a passive particle or an active swimmer, with an arbitrary shape in a linear background flow at low Reynolds number, and derive a generalized version of the Jeffery equations for the angular dynamics of the object, including a new constant from the chirality of the object as well as the Bretherton constant. The new constant appears from the inhomogeneous chirality distribution along the axis of the helicoidal symmetry, whereas the overall chirality of the object contributes to the drift velocity. Further investigations are made for an object in a simple shear flow, and it is found that the chirality effects generate non-closed trajectories of the director vector which will be stably directed parallel or anti-parallel to the background vorticity vector depending on the sign of the chirality. A bacterial swimmer is considered as an example of a helicoidal object, and we calculate the values of the constants in the generalized Jeffery equations for a typical morphology of Escherichia coli. Our results provide useful expressions for the studies of microparticles and biological fluids, and emphasize the significance of the symmetry of an object on its motion at low Reynolds number.

Key words: micro-organism dynamics

\section{Introduction}

When we observe fluid flow, we sometimes perceive its motion via the motions of objects immersed in the fluid, e.g. bubbles in a stream, clouds in the sky and tracer particles in flow visualizations. The motions of a spherical microparticle under a background flow are governed by Stokes' law (Stokes 1851; Maxey \& Riley 1983). In the physical and biological world, however, particles immersed in a fluid are not always spheres.

The simplest shape among non-spherical particles would be a spheroid, which possesses an axis of continuous rotational symmetry, and the motions of such a particle in simple shear flow under the assumption of negligible inertia have been analytically solved (Jeffery 1922). In particular, the director vector of a spheroid 
traces a closed orbit, which is known as the Jeffery orbit, and the equations for the time evolution of the director vector are referred to as the Jeffery equations (Crowdy 2016). These angular dynamics have been widely used in the research of fluid mechanics, including studies of the rheology of suspension (Hinch \& Leal 1972; Petrie 1999; Mueller, Llwwelln \& Mader 2010) and particle motions in turbulence (Voth \& Soldati 2017).

The analytic results of these studies are very useful, and thus spheroidal swimmers have been used for models of micro-organisms and other self-propelling particles or swimmers, such as bioconvection (Pedley \& Kessler 1992; Bearon, Hazel \& Thron 2011), swimmer-swimmer and swimmer-wall hydrodynamic interactions (Spagnolie \& Lauga 2012), and swimmers in background flow (Zöttl \& Stark 2013; Kantsler et al. 2014; Ishimoto 2017; Clifton, Bearon \& Bees 2018; Walker et al. 2018).

The closed orbit of the particle director vector was first obtained by Jeffery (1922) for a spheroid in a simple shear, and Bretherton (1962) later extended these results and found that the motion of an arbitrary body of revolution in a shear is identical to that of a spheroid, with his explanations further reinforced by Brenner (1964b), who did not use the word axisymmetry for the body of revolution. From the hydrodynamics point of view, a body of revolution is not only the object whose motions are specified by the direction of the axis of symmetry, but also an object in a class of symmetry known as helicoidal symmetry, which is defined by the invariance of the hydrodynamic resistance tensors under the $\pi / 2$-rotation around an axis (Brenner 1964a,b). We call the object with this symmetry a helicoidal object.

A helix is well described as a helicoidal object. The use of helicoidal symmetry has been introduced as a unidirectional approximation for the motion of rigid helices by Chen \& Zhang (2011) and as an axisymmetric propulsion in terms of bacterial swimming by Ishimoto (2019) and Ishimoto \& Lauga (2019). Chen \& Zhang (2011) performed numerical computations and found that the variation in the components of resistive matrices remains less than $1 \%$ during one cycle of the rotation of a helix with a bacterial morphology. The validity of the symmetric reduction is also provided by Kim \& Rae (1991) who studied a rigid helix in a shear flow and compared the computations from the slender-body theory with the azimuthal average approximation.

The motions of a chiral object such as a helix in a background flow have been intensively studied in the last decade, and the coupling between the chirality and the external fluid flow has been found to generate an additional drift force, which enables us to sort the objects by the use of flow in a microdevice (Doi \& Makino 2005; Makino \& Doi 2005; Marcos et al. 2009; Eichhorn 2010; Chen \& Zhang 2011; Aristov, Eichhorn \& Bechinger 2013; Hermans et al. 2015; Ro, Yi \& Kim 2016). This hydrodynamic coupling for a chiral object also leads to biased locomotion of the bacteria in a shear flow (Marcos et al. 2012) and preferential rotation of a particle in three-dimensional homogeneous isotropic turbulence (Kramel et al. 2015).

Extensions of the Jeffery orbits have also been made for chiral objects by Chen \& Zhang (2011), who studied the motion of a chiral rod-like object such as DNA and chiral disk hexamers and proved that the director dynamics are identical to the Jeffery orbit but with additional drift motion. The symmetry class of 'isotropic helicoids', which possess a triaxial helicoidal symmetry, has also been studied, and the director dynamics are the same as that of a simple sphere (Gustavsson \& Biferale 2016).

Kramel et al. (2015) studied a chiral rod-like object with two connected helices of opposite chirality, and they found the preferential rotation of a helicoidal particle in three-dimensional homogeneous isotropic turbulence. They also proposed a mathematical model that explains the biased orientation, considering additional 
rotational dynamics induced by the chirality-related hydrodynamic coupling. However, note that the chirality effects were empirically introduced, and their particles possessed additional fore-aft symmetry. Marcos et al. (2012) studied the locomotion of bacteria in a shear flow. Bacteria can swim with a helical filamentous appendage, known as a flagellum, rotated by a molecular motor at the base end of the flagellum. They found that the bacterium tends to swim toward the background vorticity vector via the hydrodynamic torque originating from the coupling between the bacterial flagellar chirality and the background flow. These studies have shown the breakdown of the Jeffery-type closed orbit by chirality. However, to the best of the author's knowledge, no general theories have been provided for the dynamics of chiral objects and swimmers that provide a biased orientation in a background flow.

The aim of this paper is thus to provide an equation for the director vector of a chiral object, considering a general shape with a particular symmetry - helicoidal symmetry - and theoretically demonstrate how the biased orientation emerges. We also consider a self-propelling bacterial swimmer as an example of a general helicoidal object. However, the theoretical results of this paper are, of course, not limited to a bacterial swimmer but applicable to any type of particle and swimmer under the same symmetrical group.

The contents of the remainder of this paper are as follows. In $\S 2$, we discuss our problem setting and the general formulation of the motion of an object immersed in a linear background flow. We also introduce helicoidal symmetry and discuss the reduction of the degrees of freedom with additional symmetry. In $\S 3$, we solve the general equations of motion and derive the equations of motion for the director vector $\boldsymbol{d}$,

$$
\dot{d}=\boldsymbol{\Omega}^{\infty} \times \boldsymbol{d}+\beta(I-\boldsymbol{d} d) \cdot E^{\infty} \cdot \boldsymbol{d}+\alpha\left[(I-d \boldsymbol{d}) \cdot \boldsymbol{E}^{\infty} \cdot \boldsymbol{d}\right] \times \boldsymbol{d},
$$

where $\boldsymbol{\Omega}^{\infty}$ and $\boldsymbol{E}^{\infty}$ are the vorticity vector and the rate-of-strain tensor of the background flow, respectively, and $\boldsymbol{I}$ is the identity tensor. The first two terms of the right-hand side of (1.1) are identical to the Jeffery equation. The constant $\beta$ reflects the shape of the object and is known as the Bretherton constant (Kim \& Karrila 2005). The last term appears from the chirality of the object and $\alpha$ is a new constant; thus, equation (1.1) can be referred to as a generalized version of the Jeffery equations. We also derive the drift velocity and consider the dynamics of a helicoidal object with an additional symmetry to discuss how the Jeffery orbits should be extended. Section 4 is devoted to a detailed analysis for the case of a simple shear, and the extended version of the Jeffery orbits for a helicoidal object will be discussed. In $\S 5$, we consider a model bacterial swimmer as an example of a helicoidal object to consider the biased locomotion of an bacterial swimmer in a shear flow before proceeding to $\S 6$, where additional discussions and future perspectives will be made.

\section{Hydrodynamics and symmetry}

\subsection{Problem setting}

We consider the dynamics of a microscale object under a linear shear background flow. Owing to the small size of the object, the fluid velocity field, $\boldsymbol{u}$, is well described by the Stokes equation,

$$
\mu \nabla^{2} \boldsymbol{u}=\nabla p
$$

where the fluid velocity obeys the incompressible condition, $\boldsymbol{\nabla} \cdot \boldsymbol{u}=0$, and $p$ is the pressure field and $\mu$ is the viscosity constant. 


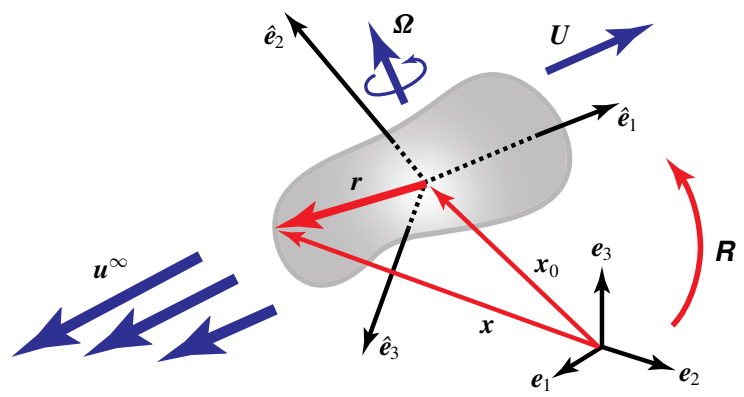

FIGURE 1. Schematic of an object with an arbitrary shape under a linear shear flow. We consider the laboratory-fixed reference frame $\left\{\boldsymbol{e}_{i}\right\}$, and the body-fixed frame $\left\{\hat{\boldsymbol{e}}_{i}\right\}$ whose origin is denoted by $\boldsymbol{x}_{0}$. The matrix $\boldsymbol{R}$ represents the rotation from the laboratory frame to the body-fixed frame. The vector $\boldsymbol{r}$ represents the relative position to the origin $\boldsymbol{x}_{0}$. The linear and angular velocities of the object are $\boldsymbol{U}$ and $\boldsymbol{\Omega}$, respectively. The background linear flow field is indicated by $\boldsymbol{u}^{\infty}$.

The no-slip boundary condition is imposed on the surface of the object, $S$, where the fluid velocity coincides with the velocity of the object $v$. We allow the object to self-propel by deformation, and then the velocity of the object is decomposed into the linear and angular velocities, $\boldsymbol{U}$ and $\boldsymbol{\Omega}$ and the deformation velocity, $\boldsymbol{u}^{\prime}$ (Yariv 2006; Ishimoto \& Yamada 2012). We introduce the two right-handed coordinate frames, the laboratory frame $\left\{\boldsymbol{e}_{i}\right\}(i=1,2,3)$ and the body-fixed frame $\left\{\hat{\boldsymbol{e}}_{i}\right\}(i=1,2,3)$ whose origin is denoted by $\boldsymbol{x}_{0}$ (figure 1 ). Thus the no-slip boundary condition on $S$ reads as

$$
\boldsymbol{u}(\boldsymbol{x})=\boldsymbol{U}+\boldsymbol{\Omega} \times \boldsymbol{r}+\boldsymbol{u}^{\prime} \text { on } S,
$$

where $\boldsymbol{r}=\boldsymbol{x}-\boldsymbol{x}_{0}$. The linear velocity, $\boldsymbol{U}$, is defined by $\boldsymbol{U}=\mathrm{d} \boldsymbol{x}_{0} / \mathrm{d} t$, and the angular velocity, $\boldsymbol{\Omega}$, is defined by using the rotation from the reference frame to the body-fixed frame as $\mathrm{d} \boldsymbol{R} / \mathrm{d} t=\boldsymbol{\Omega} \times \boldsymbol{R}$, where $\boldsymbol{R}$ is the rotation matrix from the laboratory frame to the body-fixed frame.

The background flow field, $\boldsymbol{u}^{\infty}$, is given in the absence of the object, and we assume a linear flow, which satisfies the Stokes equations. The fluid velocity around the object immersed in the fluid approaches the background flow in the far field, which gives another boundary condition for the Stokes equation (2.1):

$$
\boldsymbol{u}(\boldsymbol{x}) \rightarrow \boldsymbol{u}^{\infty}=\boldsymbol{U}^{\infty}+\boldsymbol{\Omega}^{\infty} \times \boldsymbol{r}+\boldsymbol{r} \cdot \boldsymbol{E}^{\infty}, \quad \text { as } \boldsymbol{x} \rightarrow \infty .
$$

Here, $\boldsymbol{U}^{\infty}$ is the background linear velocity, and $\boldsymbol{\Omega}^{\infty}$ is the background angular velocity, which is obtained from the antisymmetric part of the velocity gradient tensor (following Brenner (1964b), we define $\boldsymbol{G}=\nabla \boldsymbol{u}^{\infty}$ as $G_{i j}=\partial u_{j}^{\infty} / \partial x_{i}$, although the nabla operator is usually defined so that $[\nabla \boldsymbol{a}]_{i j}=\partial a_{i} / \partial x_{j}$ for a vector $\boldsymbol{a}$. The rest of the manuscript, however, is not affected by the change in this definition); $\boldsymbol{G}=\nabla \boldsymbol{u}^{\infty} \cdot \boldsymbol{E}^{\infty}$ is the rate-of-strain tensor from the background flow, corresponding to the symmetric part of the velocity gradient tensor, $\boldsymbol{E}^{\infty}=\left(\boldsymbol{G}+\boldsymbol{G}^{\mathrm{T}}\right) / 2$, with the superscript, $\mathrm{T}$, representing the transpose of a tensor.

The dynamics of the object with an arbitrary shape in a background shear flow are obtained by the force and torque balance equations due to the negligible inertia at low Reynolds number. From the linearity of the Stokes equations, the force and torque on 
the object are written in the form (Brenner 1964b; Kim \& Karrila 2005)

$$
\begin{gathered}
\boldsymbol{F}=\boldsymbol{K} \cdot\left(\boldsymbol{U}-\boldsymbol{U}^{\infty}\right)+\boldsymbol{C}^{\mathrm{T}} \cdot\left(\boldsymbol{\Omega}-\boldsymbol{\Omega}^{\infty}\right)+\boldsymbol{\Gamma}: \boldsymbol{E}^{\infty}+\boldsymbol{F}_{\text {prop }} \\
\boldsymbol{M}=\boldsymbol{C} \cdot\left(\boldsymbol{U}-\boldsymbol{U}^{\infty}\right)+\boldsymbol{Q} \cdot\left(\boldsymbol{\Omega}-\boldsymbol{\Omega}^{\infty}\right)+\boldsymbol{\Lambda}: \boldsymbol{E}^{\infty}+\boldsymbol{M}_{\text {prop }} .
\end{gathered}
$$

The symmetric tensors, $\boldsymbol{K}$ and $\boldsymbol{Q}$, are known as the translational and rotational tensors, respectively, and the coupling (pseudo-)tensor, $\boldsymbol{C}$, is not symmetric in general. The third-rank tensors, $\boldsymbol{\Gamma}$ and $\boldsymbol{\Lambda}$, are known as the shear-force tensor and shear-torque (pseudo-)tensor, respectively. The double dot product $\boldsymbol{\Gamma}: \boldsymbol{E}^{\infty}$ is defined as $\left[\boldsymbol{\Gamma}: \boldsymbol{E}^{\infty}\right]_{i}=$ $\Gamma_{i j k} E_{k j}^{\infty}$, where the repeated indices are summed over $j, k \in\{1,2,3\}$. These tensors are all dependent only on the shape of the object. Note that $\boldsymbol{\Gamma}$ and $\boldsymbol{\Lambda}$ can be defined without loss of generality so that they are symmetric with respect to the exchange of the second and third indices, i.e., $\Gamma_{i j k}=\Gamma_{i k j}$ and $\Lambda_{i j k}=\Lambda_{i k j}$, as the rate-of-strain tensor is symmetric. We will use a 'transpose' symbol for the exchange of the second and third indices for a third-rank tensor, which allows us to express (2.6) simply as

$$
\boldsymbol{\Gamma}=\boldsymbol{\Gamma}^{\mathrm{T}} \quad \text { and } \quad \boldsymbol{\Lambda}=\boldsymbol{\Lambda}^{\mathrm{T}} .
$$

The last terms in (2.4) and (2.5), denoted by $\boldsymbol{F}_{\text {prop }}$ and $\boldsymbol{M}_{\text {prop }}$, respectively, correspond to the propulsive force and torque generated by the deformation of the object.

For explicit forms of the tensors and propulsive force and torque, we introduce a local hydrodynamic force on the surface of the object, $\hat{f}(\boldsymbol{x})$, which is generated by a rigid motion whose velocity is given by $\hat{\boldsymbol{u}}(\boldsymbol{x})=\hat{\boldsymbol{U}}+\hat{\boldsymbol{\Omega}} \times \boldsymbol{r}$. Due to the linearity, the surface force can be decomposed into $\hat{\boldsymbol{f}}=\boldsymbol{\Sigma} \cdot \hat{\boldsymbol{U}}+\boldsymbol{\Pi} \cdot \hat{\boldsymbol{\Omega}}$ (Pozrikidis 1992), where $\boldsymbol{\Sigma}$ and $\Pi$ are the second-rank tensors known as translational and rotational surface force resistance tensors and are functions of the instantaneous object shape as other secondand third-rank tensors.

The decomposition enables us to express the propulsive force and torque (Yariv 2006; Ishimoto \& Yamada 2012) by

$$
\boldsymbol{F}_{\text {prop }}=\int_{S} \boldsymbol{u}^{\prime} \cdot \boldsymbol{\Sigma} \mathrm{d} S \text { and } \quad \boldsymbol{M}_{\text {prop }}=\int_{S} \boldsymbol{u}^{\prime} \cdot \boldsymbol{\Pi} \mathrm{d} S .
$$

The resistance tensors, $\boldsymbol{K}, \boldsymbol{C}, \boldsymbol{Q}, \boldsymbol{\Gamma}$ and $\boldsymbol{\Lambda}$, are also expressed by the translational and rotational surface force resistance tensors, and the explicit forms are summarized in appendix A. To specify the tensorial values that depend on the shape, we need six degrees of freedom for each symmetric tensor, $\boldsymbol{K}$ and $\boldsymbol{Q}$, and nine degrees of freedom for the non-symmetric tensor $\boldsymbol{C}$. Similarly, noting the relation (2.6) and the incompressibility relation $\boldsymbol{I}: \boldsymbol{E}^{\infty}=0$, where $\boldsymbol{I}$ is the identity tensor, 15 parameters represent each of the third-rank tensors $\boldsymbol{\Gamma}$ and $\boldsymbol{\Lambda}$ since the traceless condition of $\boldsymbol{E}^{\infty}$ leads to a further three gauge degrees of freedom which can be set to zero (see Brenner (1964b) for a detailed discussions). In turn, we need 51 degrees of freedom to be specified to derive the equations of motion for a particular object.

\subsection{Helicoidal symmetry}

We then follow the symmetric arguments by Brenner $(1964 a, b)$ to reduce the degrees of freedom in the resistance tensors and categorize the motions of the object, following the spirit of the hydrokinetic symmetry theory in late 19th century developed for an object in an inviscid flow (Kirchhoff 1869; Kelvin 1871; Lamor 1884; Lamb 1932). 
(a)

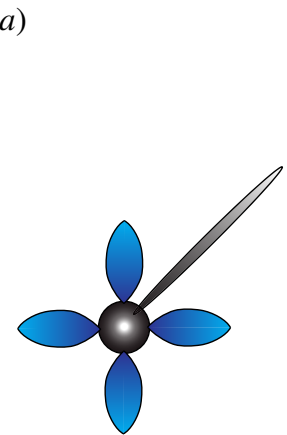

(b)
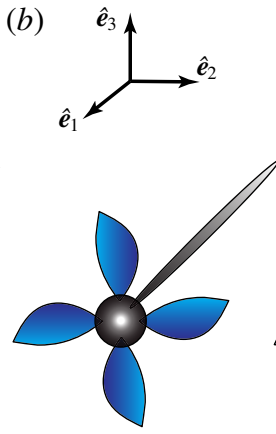

(c)

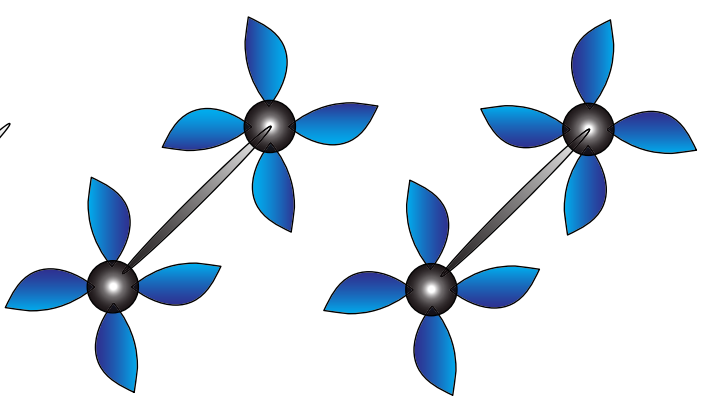

FIGURE 2. Schematics of objects with helicoidal symmetry. (a) A propeller with four symmetric blades is attached at one end of a rod. The object is symmetric in a plane including the axis of the helicoidal symmetry, and the motion is identical to that of a 'body of revolution'. (b) An asymmetric, chiral four-bladed propeller is attached at one end of a rod. (c) Asymmetric four-bladed propellers with opposite chirality are attached at the ends of a rod. The object is symmetric in a plane perpendicular to the axis of the helicoidal symmetry. (d) Asymmetric four-bladed propellers with the same chirality are attached at the ends of a rod. The object is symmetric under a $\pi$-rotation around the axis perpendicular to the axis of the helicoidal symmetry. The same four classes of symmetry are shown for a bacterial swimmer in figure 3.

Let us impose the so-called helicoidal symmetry (Brenner 1964a,b) on the shape of the object. This symmetry assumes that the resistance tensors, $\boldsymbol{K}, \boldsymbol{C}, \boldsymbol{Q}, \boldsymbol{\Gamma}$ and $\boldsymbol{\Lambda}$, are unchanged under the rotation of a coordinate system through a right angle around an axis. We choose the axis of symmetry as $\hat{\boldsymbol{e}}_{1}$ for the body-fixed coordinates (figure 2), and we write $\hat{\boldsymbol{e}}_{1}=\boldsymbol{d}$. Note that the hydrokinetic symmetry is a discrete symmetry, as the invariance of the tensors are considered under a finite angle of rotation, not an infinitesimal angle. Fries, Einarsson \& Mehlig (2017) further discuss the motions of particles with discrete symmetry.

In figure 2, some example objects with helicoidal symmetry are illustrated. These objects consist of a rod and four-bladed propellers, whose blades are asymmetric and thus chiral except for the ones used in figure $2(a)$. The objects in figure $2(c, d)$ possess two propellers at both ends of a rod, but the chiralities of the propellers are opposite. The symmetry of the object in figure $2(b)$ is minimal for a helicoidal object, as the other three objects in figure 2 possess an extra symmetry; the object (a) has reflectional symmetry in the $\hat{\boldsymbol{e}}_{1}-\hat{\boldsymbol{e}}_{2}$ plane, the object (c) also has reflectional symmetry in a plane perpendicular to the $\hat{\boldsymbol{e}}_{1}$ axis, and the object $(d)$ has $\pi$-rotational symmetry around the $\hat{\boldsymbol{e}}_{3}$ axis (or, equivalently, the $\hat{\boldsymbol{e}}_{2}$ axis).

Such a reduction of motions has been frequently used for a bacterial swimmer that rotates a helicoidal flagellum for self-propulsion (Purcell 1977; Lauga et al. 2006). A helix does not rigorously possess helicoidal symmetry, but the time average over the rotation around the axis of the helix enables us to describe the dynamics with helicoidal symmetry.

The helicoidal symmetry also requires the condition that the propulsive force and torque, $\boldsymbol{F}_{\text {prop }}$ and $\boldsymbol{M}_{\text {prop }}$, are both aligned to the director vector, $\boldsymbol{d}$, since these vectors should be invariant under a $\pi / 2$-rotation around $\boldsymbol{d}$. This expression is reasonable as a time-averaged value for a self-propelling object with a helical flagellum such as a bacterium if the flagellum is attached normal to the cell surface (Chwang \& Wu 1971; Purcell 1977; Lauga et al. 2006). 
For a helicoidal swimmer, we therefore obtain $\boldsymbol{F}_{\text {prop }}=F_{0} \boldsymbol{d}$ and $\boldsymbol{M}_{\text {prop }}=M_{0} \boldsymbol{d}$, where $F_{0}$ and $M_{0}$ are, in general, time dependent. An example swimmer is a squirmer, which is propelled by the tangential surface slipping velocity, and non-zero propulsive torque can be generated by the swirling velocity modes (Ishimoto \& Gaffney 2013; Pak \& Lauga 2014; Pedley, Brumley \& Goldstein 2016). The shape of the squirmer should not necessarily be a sphere but an arbitrary shape under helicoidal symmetry.

More generally, if we describe the propulsive force and torque by the time average over the deformation period, and $\boldsymbol{F}_{\text {prop }}$ and $\boldsymbol{M}_{\text {prop }}$ are both aligned to the director vector $\boldsymbol{d}$, we can discuss the motions in the same manner. This description is validated if the time scale of the deformation of the self-propelling particle is sufficiently faster than the time scales of the change of its orientation by the background shear flow. This slow background flow condition would usually be satisfied when one focuses on the rheotactic response of a microswimmer; otherwise, the microswimmer would be swept away by the background flow (Hill et al. 2007; Kaya \& Kose 2012; Marcos et al. 2012; Kantsler et al. 2014; Ishimoto \& Gaffney 2015; Uspal et al. 2015). A direct comparison was made for model mono-flagellated microswimmers such as a mammalian sperm cell and Leishmania (Walker et al. 2018). Numerical simulations showed that the orientation of a swimmer with a spheroidal cell body and a planar sinusoidal flagellar waveform follows a Jeffery orbit within an error of the order of $10^{-2}$, which suggests that the phase-averaged descriptions over the beat could be widely used for microswimmer dynamics in a flow.

Figure 3 shows that the same four types of helicoidal objects with different symmetries for theoretical model bacteria, as shown in figure 2. The model in figure 3(a) consists of a rigid spheroidal cell body and a rod-like flagellum such as in a simplified model without spinning motions (Spagnolie \& Lauga 2012; Riley, Das \& Lauga 2018); this is a non-chiral body of revolution and possesses a reflectional symmetry to a plane containing the axis of helicoidal symmetry. A more realistic simple model is a spheroidal cell body with a helical flagellum, and the cell will spin around the axis of the flagellum as it swims toward the axis (figure $3 b$ ). The model in figure $3(c)$ has two flagella with different chiralities, where ' $L$ ' stands for a left-handed helix and ' $R$ ' stands for a right-handed helix. This model is symmetric in a plane perpendicular to the axis of the helicoidal symmetry, $\hat{\boldsymbol{e}}_{1}$, as in the model in figure $3(c)$. In figure $3(d)$, a model with two flagella of the same chirality is shown, and this is another example of a helicoidal object with additional $\pi$-rotational symmetry around the axis perpendicular to $\hat{\boldsymbol{e}}_{1}$ as in the model in figure $3(d)$.

Although we will discuss the bacterial swimmer in a later section, we first start with an arbitrary object with helicoidal symmetry to derive the equations of the dynamics in a linear background shear flow. Following the discussions of Brenner $(1964 a, b)$ and Happel \& Brenner (1983), we may reduce the number of parameters within the resistance tensors from 51 to 13 , and the explicit form of the representation with respect to the body-fixed frame is obtained as

$$
\left.\begin{array}{c}
\boldsymbol{K}=K_{1} \boldsymbol{d} \boldsymbol{d}+K_{2}(\boldsymbol{I}-\boldsymbol{d} \boldsymbol{d}), \quad \boldsymbol{Q}=Q_{1} \boldsymbol{d} \boldsymbol{d}+Q_{2}(\boldsymbol{I}-\boldsymbol{d} \boldsymbol{d}), \\
\boldsymbol{C}=C_{1} \boldsymbol{d} \boldsymbol{d}+C_{2}(\boldsymbol{I}-\boldsymbol{d} \boldsymbol{d})+C_{23}\left(\hat{\boldsymbol{e}}_{2} \hat{\boldsymbol{e}}_{3}-\hat{\boldsymbol{e}}_{3} \hat{\boldsymbol{e}}_{2}\right),
\end{array}\right\}
$$

and

$$
\begin{aligned}
\boldsymbol{\Gamma}= & \Gamma_{1} \boldsymbol{d} \boldsymbol{d} \boldsymbol{d}+\frac{\Gamma_{2}}{2}\left[(\boldsymbol{I}-\boldsymbol{d} \boldsymbol{d}) \boldsymbol{d}+\{(\boldsymbol{I}-\boldsymbol{d} \boldsymbol{d}) \boldsymbol{d}\}^{\mathrm{T}}\right] \\
& +\frac{\Gamma_{3}}{2}\left[\left(\hat{\boldsymbol{e}}_{2} \hat{\boldsymbol{e}}_{3}-\hat{\boldsymbol{e}}_{3} \hat{\boldsymbol{e}}_{2}\right) \boldsymbol{d}+\left\{\left(\hat{\boldsymbol{e}}_{2} \hat{\boldsymbol{e}}_{3}-\hat{\boldsymbol{e}}_{3} \hat{\boldsymbol{e}}_{2}\right) \boldsymbol{d}\right\}^{\mathrm{T}}\right],
\end{aligned}
$$




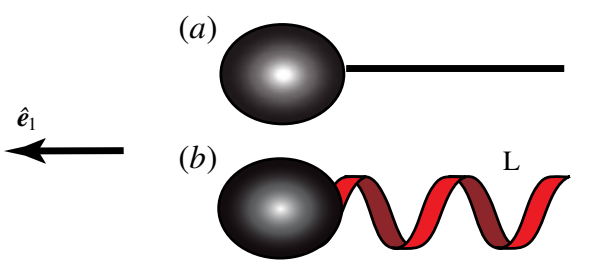

(c)

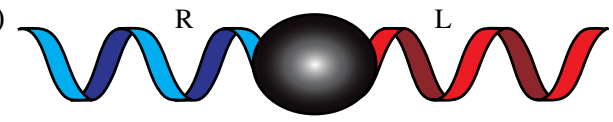

(d)

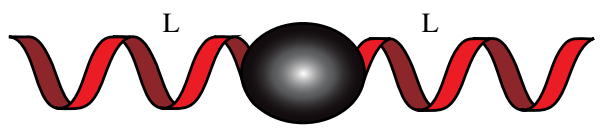

FIGURE 3. Schematics of models with helicoidal symmetry. The four symmetries are shown as in the four types of objects in figure 2. (a) A spheroidal cell body with a slender rod, which is a body of revolution with no chirality effects. (b) A spheroidal cell body with a left-handed helical flagellum. (c) A spheroidal cell body with right-handed and left-handed helical flagella. This object possesses an additional reflectional symmetry in a plane perpendicular to the axis of helicoidal symmetry, $\hat{\boldsymbol{e}}_{1} .(d)$ A spheroidal cell body with two left-handed helical flagella. This object possesses an additional $\pi$-rotational symmetry around the axis perpendicular to $\hat{\boldsymbol{e}}_{1}$.

$$
\begin{aligned}
\boldsymbol{\Lambda}= & \Lambda_{1} \boldsymbol{d} \boldsymbol{d} \boldsymbol{d}+\frac{\Lambda_{2}}{2}\left[(\boldsymbol{I}-\boldsymbol{d} \boldsymbol{d}) \boldsymbol{d}+\{(\boldsymbol{I}-\boldsymbol{d} \boldsymbol{d}) \boldsymbol{d}\}^{\mathrm{T}}\right] \\
& +\frac{\Lambda_{3}}{2}\left[\left(\hat{\boldsymbol{e}}_{2} \hat{\boldsymbol{e}}_{3}-\hat{\boldsymbol{e}}_{3} \hat{\boldsymbol{e}}_{2}\right) \boldsymbol{d}+\left\{\left(\hat{\boldsymbol{e}}_{2} \hat{\boldsymbol{e}}_{3}-\hat{\boldsymbol{e}}_{3} \hat{\boldsymbol{e}}_{2}\right) \boldsymbol{d}\right\}^{\mathrm{T}}\right],
\end{aligned}
$$

where the transpose of a triad indicates the exchange of the second and third indices as introduced before. The scalars $K_{1}, K_{2}, Q_{1}$ and $Q_{2}$ are all negative, but $C_{23}$ can be positive, negative or zero depending on the origin of the body-fixed frame. Here, $C_{1}$ and $C_{2}$ can take both signs, reflecting the chirality of the object, and they become zero when the object is non-chiral. The representations of $\boldsymbol{\Gamma}$ and $\boldsymbol{\Lambda}$ follow the expressions in Brenner (1964b), but there is a slight difference for the scalars, which become identical, after replacement such that $\Gamma_{1}=\phi_{1}^{(0)}+2 \phi_{2}^{(0)}$ and $\Lambda_{1}=\tau_{1}^{(0)}+2 \tau_{2}^{(0)}$, and $\Gamma_{i}=2 \phi_{i}^{(0)}$ and $\Lambda_{i}=2 \tau_{i}^{(0)}$, for $i=2,3$. The expressions in Chen \& Zhang (2011) are apparently similar, but they further reduce the representation assuming an additional $\pi$-rotational symmetry about an axis perpendicular to $\boldsymbol{d}$, which corresponds to the symmetry illustrated in figure $2(d)$.

The helicoidal symmetry with an additional reflectional symmetry with respect to the plane containing the $\boldsymbol{d}$ axis, as illustrated in figure 2(a), loses the chiral effects, and we have the scalars $\Gamma_{3}=\Lambda_{1}=\Lambda_{2}=0$, in addition to the scalars $C_{1}=C_{2}=0$. Thus the symmetry of this type is identical to a body of revolution, such as a spheroid, whose motions in a simple shear are characterized by a single parameter known as the Bretherton constant. The generalized helicoidal symmetry, which imposes invariance under the rotation of an arbitrary fixed angle $\theta$ around $\boldsymbol{d}$, is also identical to that for a body of revolution if the reflectional symmetry in the plane containing the $\boldsymbol{d}$ axis is further added (Brenner 1964a,b). Thus, with $N$ being any positive integer, an object with a $N$-bladed symmetric propeller attached to the end of a rod also has to follow the Jeffery orbits of any bodies of revolution.

An additional reflectional symmetry, shown in figure $2(c)$, will lead to $C_{1}=C_{2}=$ $C_{23}=0$ and $\Gamma_{1}=\Gamma_{2}=\Gamma_{3}=\Lambda_{1}=0$, and only six scalars thus remain non-zero. The $\pi$-rotational symmetry of figure $2(d)$ also reduces the number of non-zero scalars, and from similar symmetric arguments, we find $C_{23}=\Gamma_{1}=\Gamma_{2}=\Lambda_{1}=\Lambda_{2}=0$. In these two cases of additional symmetry, the helicoidal symmetry is not readily extended to the generalized version of helicoid symmetry, which imposes an invariance under the rotation of an arbitrary fixed angle $\theta$ around $\boldsymbol{d}$. The exceptional case is the 
$\pi / N$-rotational invariant around $\boldsymbol{d}$ with additional $\pi$-rotational symmetry shown in figure $2(d)$, an example being $2 N$-bladed, asymmetric chiral propellers attached to both ends of a rod in the same manner as figure $2(d)$. In this case, symmetric arguments result in the same result as the four-bladed case, where $C_{23}=\Gamma_{1}=\Gamma_{2}=\Lambda_{1}=\Lambda_{2}=0$, and the remaining eight scalars are non-zero. In other cases, we need additional scalars to represent the tensors in (2.8)-(2.10), but we will not consider this further in this study.

\section{Motions of a helicoidal object}

\subsection{Director dynamics}

We then proceed to solve the equations of motion for the object with helicoidal symmetry and derive the equations for the dynamics of the director, $\boldsymbol{d}$. As we are focusing on the time-averaged nature of the object, the 13 scalars of the resistance tensors are constant values in time.

The dynamics of the object can be obtained from the force and torque balance relations (2.4)-(2.5), and we consider that no external forces, such as gravity and magnetic forces, are applied to the object. We then rewrite the shear-induced force and torque by introducing new second-rank tensors $\boldsymbol{\Phi}$ and $\boldsymbol{\Psi}$ that satisfy $\boldsymbol{\Gamma}: \boldsymbol{E}^{\infty}=$ $\boldsymbol{\Phi} \cdot \boldsymbol{E}^{\infty} \cdot \boldsymbol{d}$ and $\boldsymbol{\Lambda}: \boldsymbol{E}^{\infty}=\boldsymbol{\Psi} \cdot \boldsymbol{E}^{\infty} \cdot \boldsymbol{d}$. The explicit forms are given by

$$
\begin{gathered}
\boldsymbol{\Phi}=\Gamma_{1} \boldsymbol{d} \boldsymbol{d}+\Gamma_{2}(\boldsymbol{I}-\boldsymbol{d} \boldsymbol{d})+\Gamma_{3}\left(\hat{\boldsymbol{e}}_{2} \hat{\boldsymbol{e}}_{3}-\hat{\boldsymbol{e}}_{3} \hat{\boldsymbol{e}}_{2}\right), \\
\boldsymbol{\Psi}=\Lambda_{1} \boldsymbol{d} \boldsymbol{d}+\Lambda_{2}(\boldsymbol{I}-\boldsymbol{d} \boldsymbol{d})+\Lambda_{3}\left(\hat{\boldsymbol{e}}_{2} \hat{\boldsymbol{e}}_{3}-\hat{\boldsymbol{e}}_{3} \hat{\boldsymbol{e}}_{2}\right) .
\end{gathered}
$$

Let us introduce a $6 \times 6$ tensor, known as the grand resistance tensor (Happel \& Brenner 1983),

$$
\mathcal{K}=\left(\begin{array}{ll}
\boldsymbol{K} & \boldsymbol{C}^{\mathrm{T}} \\
\boldsymbol{C} & \boldsymbol{Q}
\end{array}\right)
$$

and the force and torque balance equations (2.4)-(2.5), i.e., $\boldsymbol{F}=\boldsymbol{M}=\mathbf{0}$, yields

$$
\left(\begin{array}{l}
\boldsymbol{U} \\
\boldsymbol{\Omega}
\end{array}\right)=\left(\begin{array}{l}
\boldsymbol{U}^{\infty} \\
\boldsymbol{\Omega}^{\infty}
\end{array}\right)-\mathcal{K}^{-1} \cdot\left(\begin{array}{c}
\boldsymbol{\Phi} \cdot \boldsymbol{E}^{\infty} \cdot \boldsymbol{d}+\boldsymbol{F}_{\text {prop }} \\
\boldsymbol{\Psi} \cdot \boldsymbol{E}^{\infty} \cdot \boldsymbol{d}+\boldsymbol{M}_{\text {prop }}
\end{array}\right)
$$

Solving the inverse of $\mathcal{K}$ will yield the detailed expressions for the swimming velocities. This can be done using the matrix representation in the body-fixed frame, and the results are given in the form

$$
\begin{aligned}
& \boldsymbol{U}=\boldsymbol{U}^{\infty}+\boldsymbol{A} \cdot \boldsymbol{E}^{\infty} \cdot \boldsymbol{d}+\boldsymbol{U}_{\text {prop }} \\
& \boldsymbol{\Omega}=\boldsymbol{\Omega}^{\infty}+\boldsymbol{B} \cdot \boldsymbol{E}^{\infty} \cdot \boldsymbol{d}+\boldsymbol{\Omega}_{\text {prop }}
\end{aligned}
$$

The $3 \times 3$ tensors, $\boldsymbol{A}$ and $\boldsymbol{B}$ are

$$
\begin{aligned}
\boldsymbol{A}= & \Delta_{1}^{-1}\left(-Q_{1} \Gamma_{1}+C_{1} \Lambda_{1}\right) \boldsymbol{d} \boldsymbol{d}+\Delta_{2}^{-1}\left(-Q_{2} \Gamma_{2}+C_{2} \Lambda_{2}+C_{23} \Lambda_{3}\right)(\boldsymbol{I}-\boldsymbol{d} \boldsymbol{d}) \\
& +\Delta_{2}^{-1}\left(-Q_{2} \Gamma_{3}-C_{23} \Lambda_{2}+C_{2} \Lambda_{3}\right)\left(\hat{\boldsymbol{e}}_{2} \hat{\boldsymbol{e}}_{3}-\hat{\boldsymbol{e}}_{3} \hat{\boldsymbol{e}}_{2}\right) \\
\boldsymbol{B}= & \Delta_{1}^{-1}\left(C_{1} \Gamma_{1}-K_{1} \Lambda_{1}\right) \boldsymbol{d} \boldsymbol{d}+\Delta_{2}^{-1}\left(C_{2} \Gamma_{2}-C_{23} \Gamma_{3}-K_{2} \Lambda_{2}\right)(\boldsymbol{I}-\boldsymbol{d} \boldsymbol{d}) \\
& \quad+\Delta_{2}^{-1}\left(C_{23} \Gamma_{2}+C_{2} \Gamma_{3}-K_{2} \Lambda_{3}\right)\left(\hat{\boldsymbol{e}}_{2} \hat{\boldsymbol{e}}_{3}-\hat{\boldsymbol{e}}_{3} \hat{\boldsymbol{e}}_{2}\right)
\end{aligned}
$$


respectively, and the propulsive velocities, $\boldsymbol{U}_{\text {prop }}$ and $\boldsymbol{\Omega}_{\text {prop }}$ are

$$
\begin{gathered}
\boldsymbol{U}_{\text {prop }}=\Delta_{1}^{-1}\left(-Q_{1} F_{0}+C_{1} M_{0}\right) \boldsymbol{d}, \\
\boldsymbol{\Omega}_{\text {prop }}=\Delta_{1}^{-1}\left(C_{1} F_{0}-K_{1} M_{0}\right) \boldsymbol{d},
\end{gathered}
$$

where $\Delta_{1}$ and $\Delta_{2}$ are the determinants of the block matrix of the representation, given by

$$
\Delta_{1}=K_{1} Q_{1}-C_{1}^{2} \quad \text { and } \quad \Delta_{2}=K_{2} Q_{2}-C_{2}^{2}-C_{23}^{2},
$$

and both should be positive due to the negative definiteness of the grand resistance tensor $\mathcal{K}$.

The expression of the dynamics is simplified when we employ a new right-handed orthogonal basis $\left\{\boldsymbol{d}_{1}, \boldsymbol{d}_{2}, \boldsymbol{d}_{3}\right\}$ rather than the laboratory or body-fixed frame, such that

$$
d_{1}=d, \quad d_{2}=\left\{(I-d d) \cdot E^{\infty} \cdot d\right\} \times d, \quad d_{3}=(I-d d) \cdot E^{\infty} \cdot d .
$$

Note that $\boldsymbol{d}_{2}$ and $\boldsymbol{d}_{3}$ are, in general, not unit vectors, but the lengths are the same, $\left|\boldsymbol{d}_{2}\right|=\left|\boldsymbol{d}_{3}\right|$. When the director is aligned toward the vector $\boldsymbol{E}^{\infty} \cdot \boldsymbol{d}$, in other words, when the director $\boldsymbol{d}$ corresponds to one of the principal axes of $\boldsymbol{E}^{\infty}$, we cannot define the basis since $\left|\boldsymbol{d}_{2}\right|=\left|\boldsymbol{d}_{3}\right|=0$. However, as we will see below, the expansions of these vectors enable us to proceed with the calculations straightforwardly, which can be understood by the fact that the shear flow only generates motion along the director vector when $\boldsymbol{d}$ coincides with one of the principal axes.

Using the relations $(\boldsymbol{I}-\boldsymbol{d} \boldsymbol{d}) \cdot \boldsymbol{d}_{3}=\boldsymbol{d}_{3}$ and $\left(\hat{\boldsymbol{e}}_{2} \hat{\boldsymbol{e}}_{3}-\hat{\boldsymbol{e}}_{3} \hat{\boldsymbol{e}}_{2}\right) \cdot \boldsymbol{d}_{3}=\boldsymbol{d}_{2}$, together with (3.6) and (3.8), we obtain the angular velocity perpendicular to the director vector and its time evolution, $\dot{\boldsymbol{d}}=\boldsymbol{\Omega} \times \boldsymbol{d}$, as

$$
\dot{d}=\boldsymbol{\Omega}^{\infty} \times \boldsymbol{d}+\alpha \boldsymbol{d}_{2}+\beta \boldsymbol{d}_{3}
$$

where we use the 'dot' for the time derivative, and the coefficients $\alpha$ and $\beta$, which are only determined by the shape of the object, are given by

$$
\alpha=\frac{C_{2} \Gamma_{2}-C_{23} \Gamma_{3}-K_{2} \Lambda_{2}}{K_{2} Q_{2}-C_{2}^{2}-C_{23}^{2}} \quad \text { and } \quad \beta=\frac{-C_{23} \Gamma_{2}-C_{2} \Gamma_{3}+K_{2} \Lambda_{3}}{K_{2} Q_{2}-C_{2}^{2}-C_{23}^{2}} \text {. }
$$

Equations (3.13)-(3.14) are the generalized Jeffery equations for the director dynamics of a helicoidal object in a linear background flow. The non-chiral Jeffery orbit is obtained when $\alpha=0$ and $-1<\beta<1$, the latter of which corresponds to the Bretherton constant. The new coefficient $\alpha$ appears from chirality effects, and the objects no longer follow the Jeffery orbits, yielding a nonlinearity in the director dynamics (3.13). In contrast, the Jeffery equations of the non-chiral object when $\alpha=0$ are still linear in $\boldsymbol{d}$, and thus, the general motions are obtained from the analysis of the eigenvalues and eigenvectors (Bretherton 1962).

The generalized Jeffery equations (3.13)-(3.14) do not seem to include the active propulsive effects of a swimmer, but note that the equations hold for an active microswimmer as well as a passive particle since the propulsive contributions, which are parallel to the director vector, are decoupled from the angular dynamics. 


\subsection{Motion of an object}

We then proceed to consider the drift velocity, the linear velocity perpendicular to the director vector, which is obtained from (3.5) and (3.7) as

$$
\begin{aligned}
\left(\boldsymbol{U}-\boldsymbol{U}^{\infty}\right) \cdot(\boldsymbol{I}-\boldsymbol{d} \boldsymbol{d})= & \Delta_{2}^{-1}\left(-Q_{2} \Gamma_{3}-C_{23} \Lambda_{2}+C_{2} \Lambda_{3}\right) \boldsymbol{d}_{2} \\
& +\Delta_{2}^{-1}\left(-Q_{2} \Gamma_{2}+C_{2} \Lambda_{2}+C_{23} \Lambda_{3}\right) \boldsymbol{d}_{3}
\end{aligned}
$$

from which we find there is, in general, a non-zero drift motion by the linear background shear flow. Similarly, the dynamics along the director vector are also obtained from (3.5) to (3.10). Direct computations readily show that

$$
\begin{gathered}
\boldsymbol{U} \cdot \boldsymbol{d} \boldsymbol{d}=\boldsymbol{U}^{\infty} \cdot \boldsymbol{d} \boldsymbol{d}+\Delta_{1}^{-1}\left(-Q_{1} \Gamma_{1}+C_{1} \Lambda_{1}\right) \boldsymbol{E}^{\infty}: \boldsymbol{d} \boldsymbol{d} \boldsymbol{d}+\boldsymbol{U}_{p r o p} \\
\boldsymbol{\Omega} \cdot \boldsymbol{d} \boldsymbol{d}=\boldsymbol{\Omega}^{\infty} \cdot \boldsymbol{d} \boldsymbol{d}+\Delta_{1}^{-1}\left(C_{1} \Gamma_{1}-K_{1} \Lambda_{1}\right) \boldsymbol{E}^{\infty}: \boldsymbol{d} \boldsymbol{d} \boldsymbol{d}+\boldsymbol{\Omega}_{\text {prop }}
\end{gathered}
$$

which contain a non-trivial velocity contribution from the shear in addition to the selfpropulsive velocities. This is in contrast to the drift velocity, which does not include self-propulsive contributions.

For further clarification of the origins of the non-trivial velocity contributions, we consider the reductions by an additional symmetry, as illustrated in figures $2(a, c, d)$ and $3(a, c, d)$.

\subsubsection{Helicoidal objects with reflectional symmetry in a plane containing the helix axis}

With this additional symmetry, the object loosens the chiral effects, and we have $C_{1}=C_{2}=\Gamma_{3}=\Lambda_{1}=\Lambda_{2}=0$. The schematics are shown in figures $2(a)$ and $3(a)$. The coefficients in the director equations (3.13) are then reduced to

$$
\alpha=0 \quad \text { and } \quad \beta=\frac{K_{2} \Lambda_{3}-C_{23} \Gamma_{2}}{K_{2} Q_{2}-C_{23}^{2}},
$$

which therefore show that the object traces the Jeffery orbits with the corresponding Bretherton constant if a simple linear shear is applied. The drift velocity (3.15) becomes

$$
\left(\boldsymbol{U}-\boldsymbol{U}^{\infty}\right) \cdot(\boldsymbol{I}-\boldsymbol{d} \boldsymbol{d})=\frac{-Q_{2} \Gamma_{2}+C_{23} \Lambda_{3}}{K_{2} Q_{2}-C_{23}^{2}} \boldsymbol{d}_{3},
$$

which has a non-zero value. If we consider a simple linear shear, where the object periodically rotates following the Jeffery orbits, the time average of the vector $\boldsymbol{d}_{3}$ over the period of a closed orbit then becomes zero, or $\left\langle\boldsymbol{d}_{3}\right\rangle=\mathbf{0}$, with the time average denoted by a bracket \langle\rangle . Thus the net drift velocity vanishes, $\boldsymbol{U}_{\text {drift }}=\left\langle\left(\boldsymbol{U}-\boldsymbol{U}^{\infty}\right) \cdot\right.$ $(\boldsymbol{I}-\boldsymbol{d} \boldsymbol{d})\rangle=\mathbf{0}$. The linear and angular velocity components toward the director vector are obtained from (3.16) to (3.17), as

$$
\begin{gathered}
\left(\boldsymbol{U}-\boldsymbol{U}^{\infty}\right) \cdot \boldsymbol{d} \boldsymbol{d}=-\frac{\Gamma_{1}}{K_{1}} \boldsymbol{E}^{\infty}: \boldsymbol{d} \boldsymbol{d} \boldsymbol{d}-\frac{F_{0}}{K_{1}} \boldsymbol{d}, \\
\left(\boldsymbol{\Omega}-\boldsymbol{\Omega}^{\infty}\right) \cdot \boldsymbol{d} \boldsymbol{d}=-\frac{M_{0}}{Q_{1}} \boldsymbol{d} .
\end{gathered}
$$


3.2.2. Helicoidal objects with reflectional symmetry in a plane perpendicular to the helix axis

Similar to the above case, some scalars vanish and we have $C_{1}=C_{2}=C_{23}=\Gamma_{1}=$ $\Gamma_{2}=\Gamma_{3}=\Lambda_{1}=0$. The schematics are shown in figures $2(c)$ and $3(c)$. The coefficients in the director equations are

$$
\alpha=-\frac{\Lambda_{2}}{Q_{2}} \quad \text { and } \quad \beta=\frac{\Lambda_{3}}{Q_{2}} .
$$

The non-zero value of $\alpha$ therefore indicates the breakdown of the Jeffery orbits for this type of object. The drift velocity, however, vanishes, since

$$
\left(\boldsymbol{U}-\boldsymbol{U}^{\infty}\right) \cdot(\boldsymbol{I}-\boldsymbol{d} \boldsymbol{d})=\mathbf{0}
$$

from (3.15). The linear and angular velocities toward the director vector are also obtained as

$$
\left(\boldsymbol{U}-\boldsymbol{U}^{\infty}\right) \cdot \boldsymbol{d}=-\frac{F_{0}}{K_{1}} \quad \text { and } \quad\left(\boldsymbol{\Omega}-\boldsymbol{\Omega}^{\infty}\right) \cdot \boldsymbol{d}=-\frac{M_{0}}{Q_{1}},
$$

which indicates that only the propulsive linear and angular velocities appear.

\subsubsection{Helicoidal objects with $\pi$-rotational symmetry around an axis perpendicular to the helix axis}

This symmetry corresponds to the schematics in figures $2(d)$ and $3(d)$. The director dynamics are reduced by the vanishing scalars, $C_{23}=\Gamma_{1}=\Gamma_{2}=\Lambda_{1}=\Lambda_{2}=0$, and we have the coefficients

$$
\alpha=0 \quad \text { and } \quad \beta=\frac{K_{2} \Lambda_{3}-C_{2} \Gamma_{3}}{K_{2} Q_{2}-C_{2}^{2}}
$$

which again shows that the object should follow the Jeffery orbits if it is immersed in a simple linear shear, as obtained by Chen \& Zhang (2011). The drift velocity is calculated as

$$
\left(\boldsymbol{U}-\boldsymbol{U}^{\infty}\right) \cdot(\boldsymbol{I}-\boldsymbol{d} \boldsymbol{d})=\frac{-Q_{2} \Gamma_{3}+C_{2} \Lambda_{3}}{K_{2} Q_{2}-C_{2}^{2}} \boldsymbol{d}_{2},
$$

which reflects the chirality, as the scalar $C_{2}$ flips its sign depending on the sign of the chirality. When the object is in a simple linear shear and exhibits a periodic director motion as in the Jeffery orbit, the drift velocity yields a non-zero value after the time average over the period of rotation, and the direction of the drift motion is parallel or anti-parallel to the background vorticity vector. Finally, we show the expressions for the linear and angular velocities along the director vector:

$$
\left(\boldsymbol{U}-\boldsymbol{U}^{\infty}\right) \cdot \boldsymbol{d}=\frac{-Q_{1} F_{0}+C_{1} M_{0}}{K_{1} Q_{1}-C_{1}^{2}} \quad \text { and } \quad\left(\boldsymbol{\Omega}-\boldsymbol{\Omega}^{\infty}\right) \cdot \boldsymbol{d}=\frac{C_{1} F_{0}-K_{1} M_{0}}{K_{1} Q_{1}-C_{1}^{2}},
$$

which simply correspond to the propulsive velocities. 
(a)

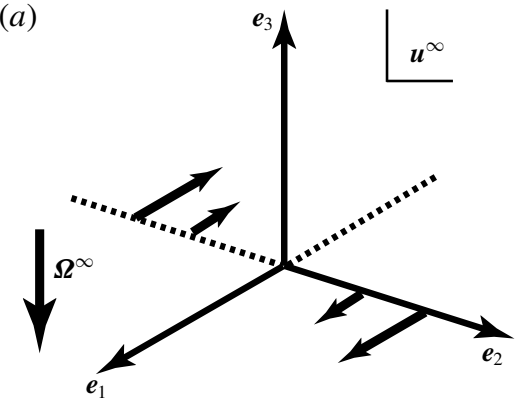

(b)

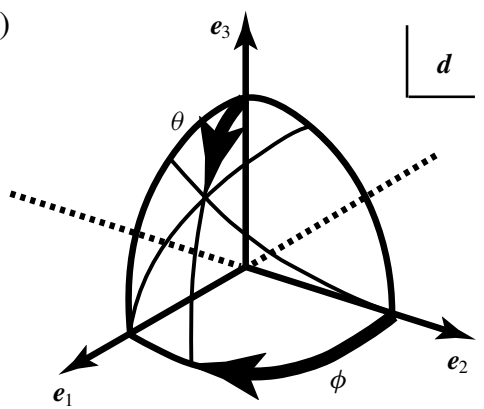

FIGURE 4. (a) Schematics of a background simple shear flow and $(b)$ the angle variables for the director vector. (a) A simple shear is considered, $\boldsymbol{u}^{\infty}=\gamma y \boldsymbol{e}_{1}$, where $\gamma \geqslant 0$ is the shear strength. The background vorticity vector is then directed to the negative $\boldsymbol{e}_{3}$ axis. (b) The director dynamics are parameterized by the two angles, the polar angle $\theta \in[0, \pi]$ measured from the $\boldsymbol{e}_{3}$ axis and the azimuthal angle $\phi \in(-\pi, \pi]$ measured from the $\boldsymbol{e}_{2}$ axis toward the $\boldsymbol{e}_{1}$ axis.

\section{Dynamics in a simple shear}

In this section, we proceed to the director dynamics (3.13) in a simple linear shear to compare it with the Jeffery orbits. We assume that the background flow $\boldsymbol{u}^{\infty}$ is a simple shear given by $\boldsymbol{u}^{\infty}=\gamma y \boldsymbol{e}_{1}$ with shear strength $\gamma \geqslant 0$ as schematically shown in figure $4(a)$. The background vorticity vector is $\boldsymbol{\Omega}^{\infty}=-(\gamma / 2) \boldsymbol{e}_{3}$, which is directed toward the $-\boldsymbol{e}_{3}$ axis. The trajectories of a particle or a swimmer are, in general, not simple, since the equations only provide the instantaneous time-dependent velocity, and the background flow depends on the instantaneous position. We thus hereafter focus on the director dynamics of a helicoidal object.

\subsection{Angle dynamics}

The director dynamics can be described by the two angle variables, for which we use the polar and azimuthal angles as shown in figure $4(b)$. The director vector, $\boldsymbol{d}=$ $d_{x} \boldsymbol{e}_{1}+d_{y} \boldsymbol{e}_{2}+d_{z} \boldsymbol{e}_{3}$, is parameterized as

$$
d_{x}=\sin \theta \sin \phi, \quad d_{y}=\sin \theta \cos \phi \quad \text { and } \quad d_{z}=\cos \theta .
$$

After some mathematical manipulation, we may rewrite (3.13) as

$$
\begin{gathered}
\dot{d}_{x}=\gamma\left[\frac{d_{y}}{2}+\beta\left(\frac{d_{y}}{2}-d_{x}^{2} d_{y}\right)+\frac{\alpha}{2} d_{x} d_{z}\right], \\
\dot{d}_{y}=\gamma\left[-\frac{d_{x}}{2}+\beta\left(\frac{d_{x}}{2}-d_{x} d_{y}^{2}\right)-\frac{\alpha}{2} d_{y} d_{z}\right], \\
\dot{d}_{z}=\gamma\left[-\beta d_{x} d_{y} d_{z}+\frac{\alpha}{2}\left(d_{y}^{2}-d_{x}^{2}\right)\right] .
\end{gathered}
$$

By substituting the expressions (4.1) into the above equations, we finally obtain the ordinary differential equations for the angle variables as

$$
\frac{\mathrm{d} \theta}{\mathrm{d} t}=\gamma\left(\frac{\beta}{4} \sin 2 \theta \sin 2 \phi-\frac{\alpha}{2} \sin \theta \cos 2 \phi\right),
$$




$$
\frac{\mathrm{d} \phi}{\mathrm{d} t}=\gamma\left(\frac{1}{2}+\frac{\beta}{2} \cos 2 \phi+\frac{\alpha}{2} \cos \theta \sin 2 \phi\right) .
$$

The shear strength only changes the time scale of the dynamics, and we hereafter fix $\gamma=1$. When $\alpha=0$, we readily obtain the angle dynamics of the periodic motion known as the Jeffery orbits since the $\phi$ dynamics are integrable. However, in the general case when $\alpha \neq 0$, the angle variables are coupled, and the director dynamics become more complicated.

Another extreme case of periodic motion is obtained when $\beta=0$. The equations (4.5)-(4.6) are integrable, and the integral curve is given by

$$
\alpha \sin 2 \phi \sin ^{2} \theta-2 \cos \theta=\text { const. }
$$

In figure 5, the trajectories of the angles in the phase space are shown for several sets of parameters, $\alpha$ and $\beta$. The parameter values associated with figure 5 are plotted in the $\beta-\alpha$ plane of figure 6 , where the structures of the dynamical systems of (4.5)-(4.6) are summarized, although their detailed analysis is postponed until $\S 4.2$. Hereafter, we consider a non-negative $\alpha \geqslant 0$, since the dynamics with negative $\alpha$ are identical after a change in the variable $\theta \mapsto \pi-\theta$, as readily found from (4.5)-(4.6).

When $\alpha=0$, periodic Jeffery orbits are obtained (figure $5 a$ ). By the definition of the angle $\phi$ (figure $4 b$ ), the object rotates around the background vortex vector $\boldsymbol{\Omega}^{\infty}$ with an increase of $\phi$ as time progresses. With the additional $\alpha$ term, the orientation of the chiral object is biased depending on the sign of $\alpha$, as shown in figure $5(b)$, where the parameters $(\beta, \alpha)=(0.8,0.2)$ are used. We find from the figure that the angle $\theta$ approaches $\theta \approx \pi$, where the director vector is aligned toward the background vortex vector $\boldsymbol{\Omega}^{\infty}$. In this particular example, an object with an initial angle of $\theta_{\text {init }}=\pi / 4$ reaches the value of $\theta \approx 0.8 \pi$ after a $2 \pi$-rotation of $\phi$.

Closed orbits are also obtained when $\beta=0$, and an example case is shown in figure $5(c)$, with the values of parameters $(\beta, \alpha)=(0,0.5)$. The non-zero values of $\alpha$ and $\beta$ lead to biased director dynamics, though the negative sign of $\beta$ causes the director vector to approach $\theta \approx 0$, which is anti-parallel to $\boldsymbol{\Omega}^{\infty}$ (figure $5 d$ ).

As discussed in detail in $\S 4.2$, equations (4.5)-(4.6) possess stationary solutions for the angles as the value of $\alpha$. In figure 5(e), the flows in the phase space are shown for parameter values $(\beta, \alpha)=(0.5,1.5)$, and we find two attracting spirals for $\theta \approx$ $0.7 \pi$ and two repelling spirals for $\theta \approx 0.3 \pi$. The director vector does not reach the point where $\theta=\pi$, which is different from the case with a smaller $\alpha$ in figure $5(b)$. Closed loops are again obtained even after the stationary solutions emerge if $\beta=0$, as shown in figure $5(f)$. This is comparable to the closed Jeffery orbits with $\alpha=0$ being possible only when $|\beta|<1$. When the parameters are $(\beta, \alpha)=(1.5,0.2)$, the spiral behaviours around the non-trivial stationary angles cease, though there are still two repelling and two attracting points (figure $5 g$ ).

In figure $5(h)$, the time evolutions of the polar angle $\theta$ are plotted for the non-periodic cases (figure $5 b, d, e, g$ ) from three different initial polar angles $\theta=$ $\pi / 4, \pi / 2,3 \pi / 4$ and the initial azimuthal angle fixed as $\phi=0$. The director vector gradually becomes parallel or anti-parallel to the background vorticity vector in the cases of figure $5(b, d)$, whereas the director vector is attracted toward a non-trivial orientation in the cases of figure $5(e, g)$. 

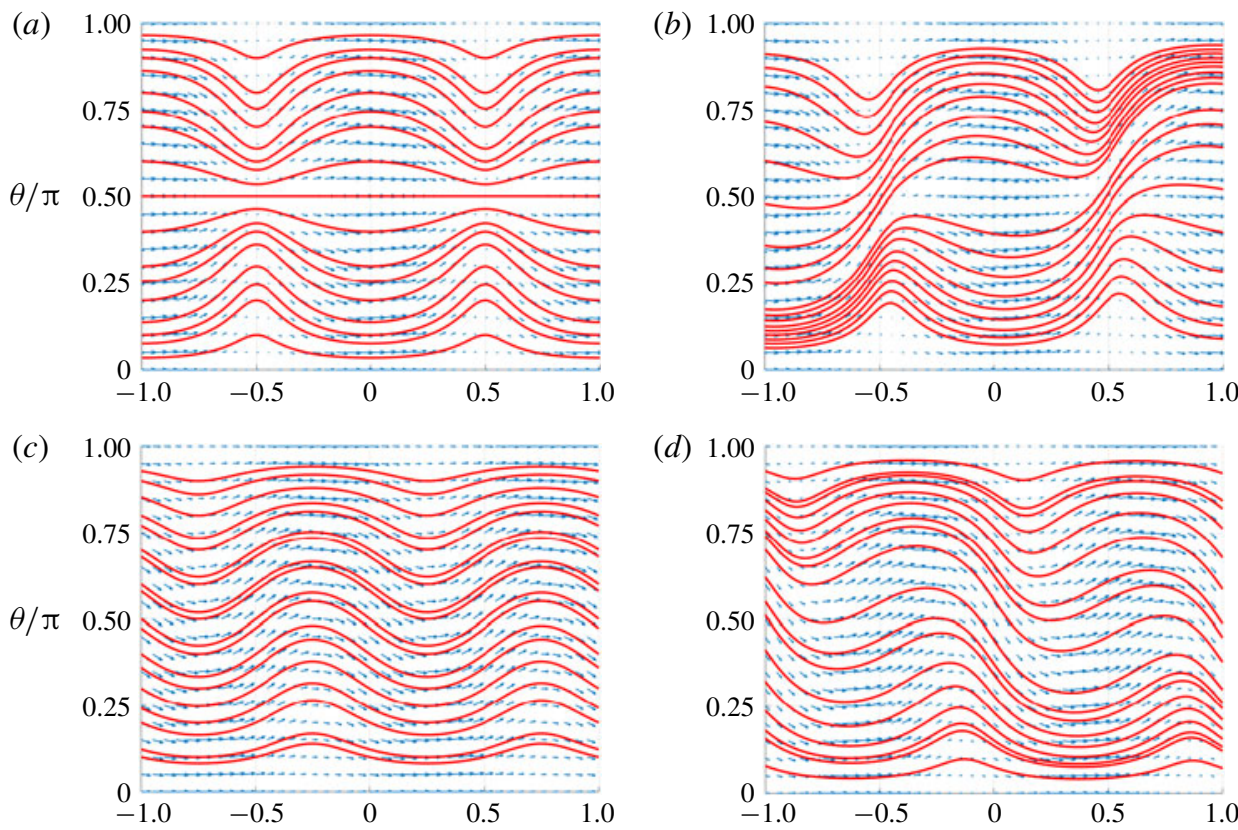

(d) 1.00
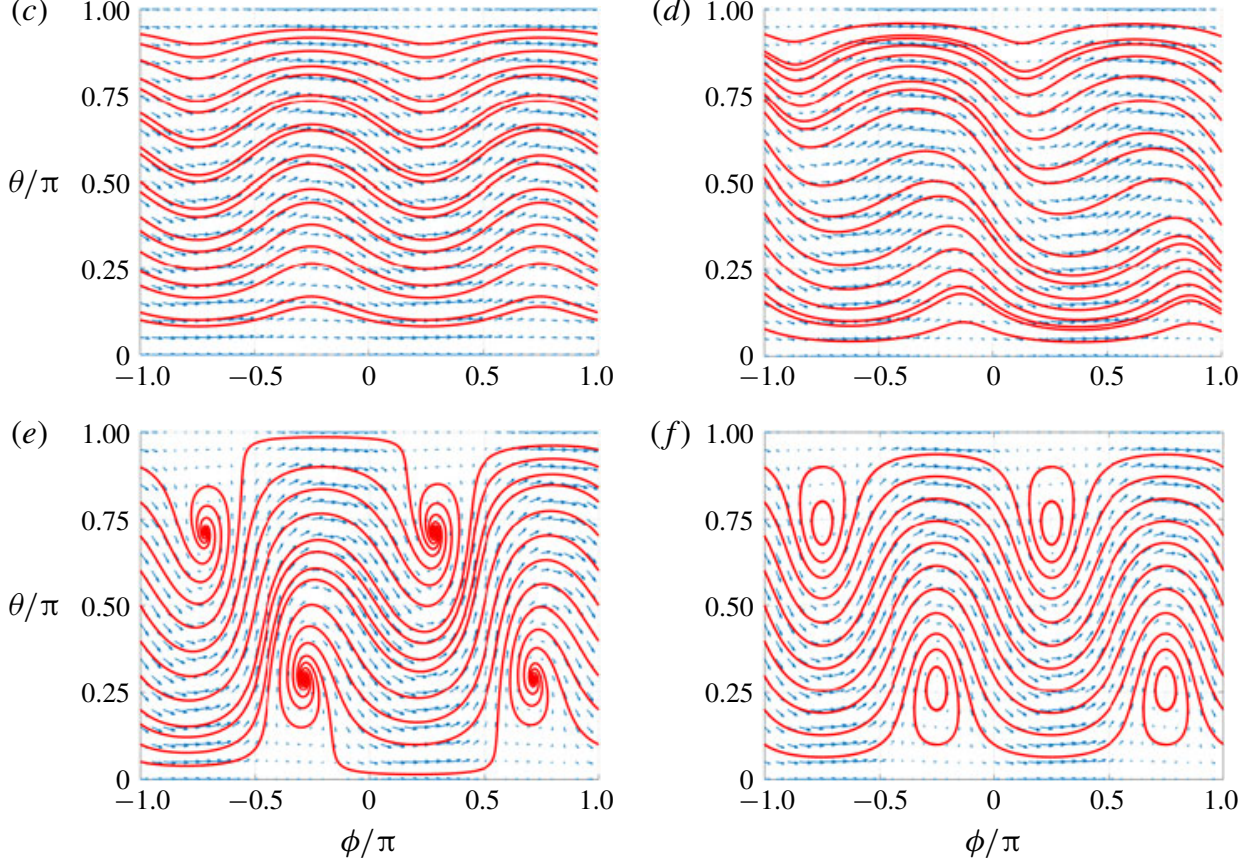

(g) 1.00

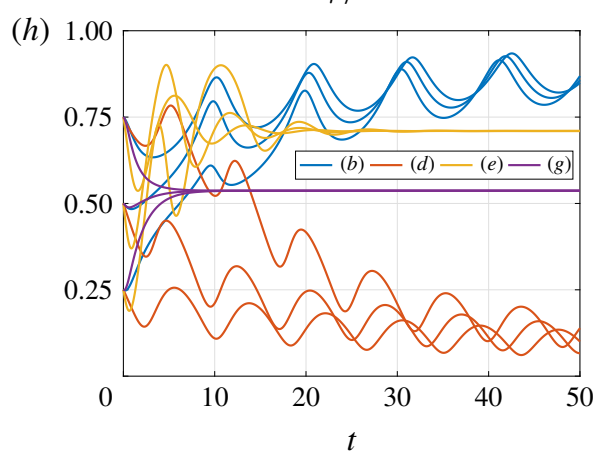

Figure 5. Angle dynamics from (4.5) to (4.6), where the red curves represent the streamline in the phase space. The blue arrows indicate the flow in the phase space. The parameters are $(a)(\beta, \alpha)=(0.8,0),(b)(\beta, \alpha)=(0.8,0.2),(c)(\beta, \alpha)=(0,0.5)$, (d) $(\beta, \alpha)=(-0.5,0.5),(e)(\beta, \alpha)=(0.5,1.5),(f)(\beta, \alpha)=(0,1.5)$ and $(g)(\beta, \alpha)=$ $(1.5,0.2)$, as plotted in red circles in figure 6. (h) Time evolution of the polar angle $\theta$ for the parameters from $(b, d, e, g)$ from three different initial polar angles $\theta=\pi / 4, \pi / 2,3 \pi / 4$ and the initial azimuthal angle fixed as $\phi=0$. 


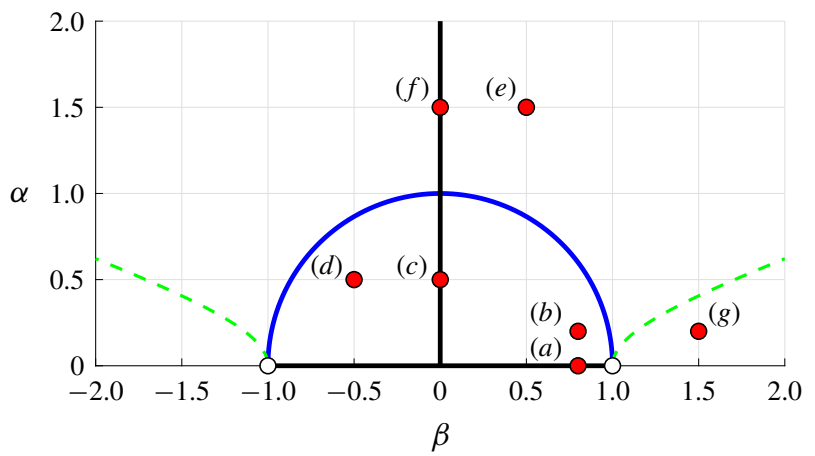

FIgURE 6. The stability diagram in the $\beta-\alpha$ plane. The black thick lines on the axes show the parameter regions, where the closed loops are obtained for the angle dynamics. The blue thick line, $\alpha^{2}+\beta^{2}=1, \alpha \neq 0$, indicates the conditions where non-trivial stationary angles bifurcate from $\theta=0, \pi$. The dashed green lines show the transition between the spiral and non-spiral dynamics around the non-trivial stationary angles, obtained from the linear stability analysis. The red circles and the associated alphabetical symbols show the parameters with which the angle dynamics are shown in figure 5. The dynamics with a negative $\alpha$ are identical to the change in the variable $\theta \mapsto \pi-\theta$ as readily found from (4.5) to (4.6).

\subsection{Some solutions and their stabilities}

We further look at the two-dimensional angle dynamics given by (4.5)-(4.6). We first consider the solutions that satisfy $\mathrm{d} \theta / \mathrm{d} t=0$. From (4.5), this is equivalent to

$$
\sin \theta(\beta \cos \theta \sin 2 \phi-\alpha \cos 2 \phi)=0,
$$

and it is readily found that $\mathrm{d} \theta / \mathrm{d} t=0$ when $\sin \theta=0$ or $\theta=0, \pi$. In this case, where the object is parallel to the vorticity vector, $\mathrm{d} \phi / \mathrm{d} t$ is always positive, and the object keeps spinning in the same direction as the background vorticity, as long as

$$
\alpha^{2}+\beta^{2}<1
$$

is satisfied. To study the stability of this periodic rotation, we expand $\theta$ around $\theta=0$, and consider the change in $\theta$ during one period of rotation, following the analysis by Kim \& Rae (1991). By denoting the period of rotation as $T$, the changes in the angle can be written as

$$
\Delta \theta=\int_{0}^{\mathrm{T}} \frac{\mathrm{d} \theta}{\mathrm{d} t} \mathrm{~d} t=\int_{0}^{2 \pi} \frac{(\mathrm{d} \theta / \mathrm{d} t)}{(\mathrm{d} \phi / \mathrm{d} t)} \mathrm{d} \phi=\frac{1}{2} \int_{0}^{2 \pi} \frac{\beta \sin 2 \theta \sin 2 \phi-2 \alpha \sin \theta \cos 2 \phi}{1+\beta \cos 2 \phi+\alpha \cos \theta \sin 2 \phi} \mathrm{d} \phi,
$$

where $\theta$, in general, depends on the value of $\phi$. To evaluate this integral we use the integral formula (Kim \& Rae 1991)

$$
\int_{0}^{2 \pi} \frac{B+C \sin 2 \phi+D \cos 2 \phi}{J+E \sin 2 \phi+F \cos 2 \phi} \mathrm{d} \phi=B P+(2 \pi-J P) \frac{C E+D F}{H^{2}}
$$


where $B, C, D, E, F$ and $J$ are constants independent of $\phi$, and $H=\sqrt{E^{2}+F^{2}}$ and $P=2 \pi / \sqrt{J^{2}-H^{2}}$. The integral in (4.10) is then estimated as

$$
\Delta \theta \simeq 2 \pi\left(1-\frac{1}{\sqrt{1-H^{2}}}\right) \frac{\alpha \beta(\sin 2 \theta \cos \theta-2 \sin \theta)}{2 H^{2}},
$$

assuming that the $\phi$-dependence of the angle $\theta$ is negligibly small. Noting that $H=$ $\alpha^{2} \cos ^{2} \theta+\beta^{2}$ and $P=2 \pi / \sqrt{1-H^{2}}$ and expanding $\theta$ around $\theta=0$, we have

$$
\Delta \theta \simeq K \frac{\alpha \beta}{\alpha^{2}+\beta^{2}} \theta^{3}
$$

where the constant $K$,

$$
K=\pi\left(\frac{1-\sqrt{1-\left(\alpha^{2}+\beta^{2}\right)}}{\sqrt{1-\left(\alpha^{2}+\beta^{2}\right)}}\right),
$$

is positive when $\alpha^{2}+\beta^{2}<1$

Thus, the change in the polar angle during one period of rotation around $\theta \approx 0$, i.e., $\Delta \theta$ in (4.13), is positive when $\alpha>0, \beta>0$ and $\alpha^{2}+\beta^{2}<1$, suggesting that the periodic motion of $\theta=0$ is unstable. Changes in the signs in $\alpha$ and $\beta$ result in changes in the stability accordingly. The change of $\theta$ during one period of rotation is proportional to $\theta^{3}$, and this very slow change of $\theta$ is compatible with the assumption of the negligible dependence of $\phi$ in integrating (4.10) and verified by direct numerical integrations of (4.5)-(4.6). Similar calculations around $\theta=\pi$ then show a positive change in the angle $\theta$ when $\alpha>0$ and $\beta>0$, corresponding to the opposite stability to the rotation around $\theta=0$. These stability analyses for the periodic rotation with $\theta=0$ and $\theta=\pi$ are also numerically confirmed by integrating equations (4.5)-(4.6).

From condition (4.8), non-trivial dynamics can arise with a stationary polar angle when $\sin \theta \neq 0$. The condition (4.8) is then rephrased to

$$
\beta \cos \theta \sin 2 \phi=\alpha \cos 2 \phi .
$$

From (4.6), the stationary azimuthal angle is obtained when $\alpha \cos \theta \sin 2 \phi+\beta \cos 2 \phi+$ $1=0$. Thus, $\mathrm{d} \theta / \mathrm{d} t=\mathrm{d} \phi / \mathrm{d} t=0$ are both satisfied if $\cos 2 \phi=-\beta /\left(\alpha^{2}+\beta^{2}\right)$. The existence condition for such an angle $\phi$ is obtained from the condition $|\cos 2 \phi| \leqslant 1$, which is equivalent to

$$
\alpha^{2}+\left(\beta \pm \frac{1}{2}\right)^{2}>\frac{1}{4} \text {. }
$$

Similarly, the existence condition for the stationary $\theta$ is obtained from (4.15), which is $|\cos \theta|=|\alpha / \beta||\cot 2 \phi| \leqslant 1$, leading to the condition

$$
\alpha^{2}+\beta^{2} \geqslant 1 \text { and } \beta \neq 0
$$

As condition (4.17) always satisfies condition (4.16), we conclude that the non-trivial stationary angles are possible when $\alpha^{2}+\beta^{2} \geqslant 1$ and $\beta \neq 0$, and these conditions are equivalent to those of non-periodic motion around $\theta=0, \pi$.

We then proceed to analyse the stability around the non-trivial stationary angles. Let $\left(\theta_{*}, \phi_{*}\right)$ be one of the non-trivial stationary angles, and from (4.15), we have $\cos 2 \phi_{*}=-\beta /\left(\alpha^{2}+\beta^{2}\right)$ and $\cos \theta_{*}= \pm \alpha / \sqrt{\left(\alpha^{2}+\beta^{2}\right)^{2}-\beta^{2}}$. We first assume $\alpha>0$ and $\beta>0$ and take one particular solution that satisfies $\pi / 2<\theta_{*}<\pi$ and $0<\phi<\pi$. 

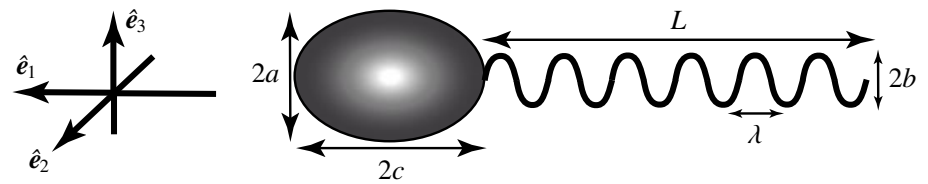

FIGURE 7. Schematics of a model bacterium with a spheroidal cell body and a helical flagellum. The cell body is a rigid spheroid with semiaxes $c, a, a$, and the flagellum is a simple circular rigid helix with pitch $\lambda$, amplitude $b$ and length $L$. The axis of the helix is set to be the swimmer's director vector, $\hat{\boldsymbol{e}}_{1}=\boldsymbol{d}$.

This solution corresponds to the stable solutions in figure $5(h)$. We then expand the dynamics (4.5)-(4.6) around these non-trivial stationary angles by introducing small angles, $\left|\theta^{\prime}\right|,\left|\phi^{\prime}\right| \ll 1$, such that $\theta=\theta_{*}+\theta^{\prime}$ and $\phi=\phi_{*}+\phi^{\prime}$. Straightforward calculations to linearize the dynamics with respect to the small angles lead to the following linear ordinary differential equations:

$$
\frac{\mathrm{d}}{\mathrm{d} t}\left(\begin{array}{l}
\theta^{\prime} \\
\phi^{\prime}
\end{array}\right)=\left(\begin{array}{ll}
A_{11} & A_{12} \\
A_{21} & A_{13}
\end{array}\right)\left(\begin{array}{l}
\theta^{\prime} \\
\phi^{\prime}
\end{array}\right),
$$

where the components are given by $A_{11}=(\beta / 2) \cos 2 \theta_{*} \sin 2 \phi_{*}-(\alpha / 2) \cos \theta_{*} \cos 2 \phi_{*}$, $A_{12}=(\beta / 2) \sin 2 \theta_{*} \cos 2 \phi_{*}+\alpha \sin \theta_{*} \sin 2 \phi_{*}, A_{21}=-(\alpha / 2) \sin \theta_{*} \sin 2 \phi_{*}$, and $A_{22}=$ $-\beta \sin 2 \phi_{*}+\alpha \cos \theta_{*} \cos 2 \phi_{*}$, respectively.

The eigenvalues of the matrix in (4.18) were numerically solved and we found that the real parts of the eigenvalues are always negative, indicating that this non-trivial stationary angle is a stable fixed point. From the components in the matrix, we then readily find that the stationary solution with $\pi / 2<\theta_{*}<\pi$ is stable and otherwise unstable when $\alpha, \beta>0$, which is compatible with the plots in $5(g, h)$. The imaginary parts can, however, be zero or non-zero, depending on the values of $\alpha$ and $\beta$, and the transition between the two cases is shown by the green dashed line in figure 6 . A spiral fixed point changes to a source or a sink when $\beta$ is large or $\alpha$ is small enough, and similar arguments follow when $\beta<0$.

The obtained structure of the dynamical system described by (4.5)-(4.6) is summarized in figure 6 . Closed orbits are possible for regions shown by the thick black lines. Within the circle of $\alpha^{2}+\beta^{2}=1, \alpha \neq 0$, depicted by the blue thick line, the orientation of the helicoidal object approaches being parallel to the background vorticity vector when $\alpha \beta>0$ and anti-parallel when $\alpha \beta<0$. Outside the blue circle, the system possesses four non-trivial stationary angles, two of which are attractive and the other two repulsive, and the orientation of the object approaches one of the attractive states.

\section{Bacterial swimmers}

We then proceed to consider a model bacterium with a spheroidal cell body and a helicoidal flagellum to obtain estimations of the parameter values of $\alpha$ and $\beta$ by use of the resistive force theory (Chwang \& Wu 1971; Lauga et al. 2006; Ishimoto 2019), following the study of the bacterial dynamics in a simple shear (Marcos et al. 2012). As shown in figure 7, we consider a spheroid of semiaxes $c, a, a$. The semiaxis of $c$ is set to be the axis of the helicoidal symmetry, $\hat{\boldsymbol{e}}_{1}$. The flagellum is simply modelled as a left-handed helix with flagellar radius $d$. We consider the radius of the helix, $b$, 
the pitch, $\lambda$, and the flagellar length along the $\hat{\boldsymbol{e}}_{1}$ axis, $L$ (figure 7 ). The cross-section of the helix is assumed to be a circle of radius $d$. We set the origin of the body-fixed frame at the centre of the spheroid.

Let $\boldsymbol{r}(s)$ be the relative position of the centreline of the flagellum from the origin of the body-fixed frame (figure 1), and let $s$ denote the arc length parameter along the flagellum. The tangent vector of the centreline is then given by $\boldsymbol{t}(s)=\mathrm{d} \boldsymbol{r} / \mathrm{d} s$. According to the resistive force theory, the hydrodynamic forces on a segment of the flagellum are linearly related to the local velocity $\boldsymbol{u}$ estimated on the centreline of the helical flagellum. The local velocity is decomposed into tangent and normal components, and we have

$$
\mathrm{d} \boldsymbol{F}(s)=\left[C_{T} \boldsymbol{t t}+C_{N}(\boldsymbol{I}-\boldsymbol{t} \boldsymbol{t})\right] \cdot \boldsymbol{u}(s) \mathrm{d} s .
$$

where $C_{T}$ and $C_{N}$ represent the tangential and normal drag coefficients, respectively, both of which are negative constants along the flagellum. We introduce the ratio of the drag coefficient, $\xi=C_{N} / C_{T}$, which is calculated from the Gray-Hancock formula (Gray \& Hancock 1955; Batchelor 1970; Tillett 1970; Rodenborn et al. 2013),

$$
C_{T}=\frac{-2 \pi \mu}{\log (2 \lambda / d)-0.5} \quad \text { and } \quad C_{N}=\frac{-4 \pi \mu}{\log (2 \lambda / d)+0.5} .
$$

We then calculated the values of $\alpha$ and $\beta$ for different bacterial morphologies, using the values of the scalars computed by the resistive force theory. The detailed expressions and derivations are found in appendix B. In figure 8, the calculated values are plotted for the model bacterium with several cell-body aspect ratios $c$, and flagellar length $L$. The parameters $a=1, \lambda=2.5$ and $b=0.25$ are fixed, and these values correspond to typical Escherichia coli bacteria (Lauga et al. 2006). In figure $8(a)$, we plot the values of $\alpha$ and $\beta$ for the choice of seven different aspect ratios for the cell body $c$. Without a flagellum, $\alpha=0$ and the values of $\beta$, given by $\beta=\left(c^{2}-1\right) /\left(c^{2}+1\right)$ (Jeffery 1922; Bretherton 1962), are marked by the blue circles on the line $\alpha=0$ in figure $8(a)$. The seven circles correspond to the values of $\beta=-0.9,-0.6, \ldots, 0.9$, which, respectively, give the aspect ratios of the cell body $c \approx 0.23, c=0.5, c \approx 0.73, c=1, c \approx 1.36, c=2$ and $c \approx 4.36$, as labelled in figure $8(a)$. The blue curves show the changes in the values as we increased $L$ from 0 to 100 . The plotted circles indicate the values for $L=0,2, \ldots, 10$ with the same colours for the same value of $L$. The same plots are shown in figure $8(b)$, but the horizontal axis is replaced by the effective aspect ratio $\tilde{c}$ which satisfies $\beta=\left(\tilde{c}^{2}-1\right) /\left(\tilde{c}^{2}+1\right)$. As the flagellar length increases the values of $\alpha$ reach a maximum and converge to 0 when $\beta$ approaches 1 , as expected from the slender-body limit.

One might be surprised that the values of $\alpha$ are relatively small, $\alpha \sim 0.01$. We then consider the time evolution of the angle $\theta$ for the bacterial swimmer with such a small $\alpha$. With a typical cell aspect ratio $c / a \sim 2-3$ and flagellar length $L \sim 7.5$ for $E$. coli (Lauga et al. 2006), from figure 8, we can estimate the values $\beta=0.9$ and $\alpha=0.005$ for a typical morphology. With these values for the coefficients, the computed dynamics of the angle $\theta$ are plotted in figure 9 , in which we show the time evolution from different initial values, $\theta_{\text {init }}=0.1 \pi, 0.2 \pi, \ldots, 0.9 \pi$ in different colours. The horizontal axis indicates the time normalized by the period of the corresponding Jeffery orbit (Jeffery 1922), $T=2 \pi\left(\tilde{c}+\tilde{c}^{-1}\right)$. Within the 10 rotations of $\phi$, the bacterial swimmer with $\theta_{\text {init }} \geqslant 0.2 \pi$ is turned toward the orientation of the vorticity vector. This bacterial turning behaviour is compatible with the experimental results (Marcos et al. 2012), in which they also used the resistive force theory for comparison. 

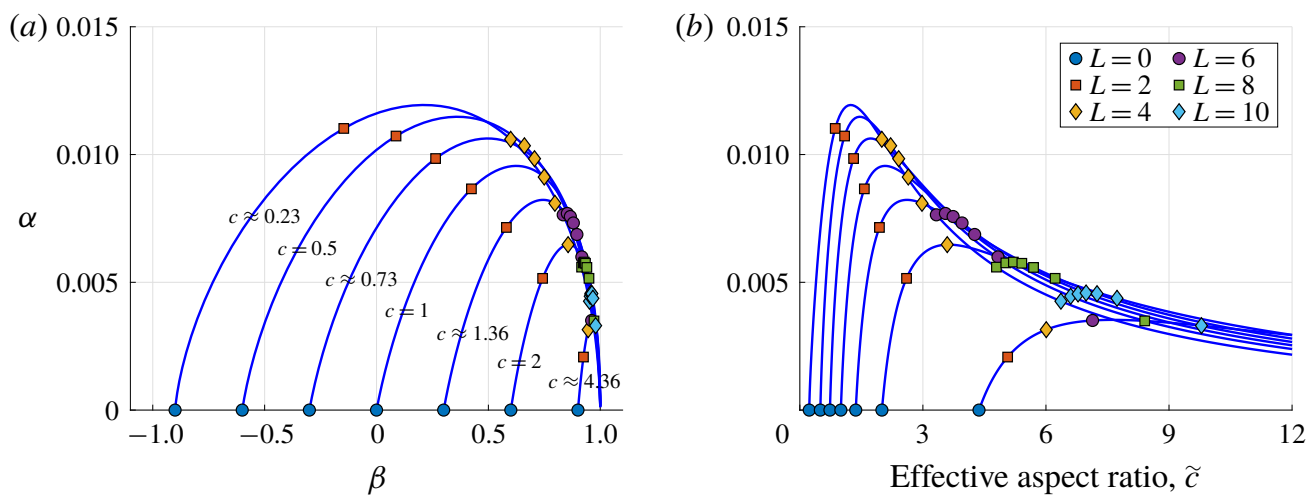

FIgURE 8. The values of $\alpha$ and $\beta$ for a model bacterium from figure 7 from the resistive force theory with various cell body aspect ratios $c$ and flagellar lengths $L$. (a) The values are plotted in the $\beta-\alpha$ plane. The seven different values of $c$ are considered, and the values used are labelled on each curve in the plot. $(b)$ The horizontal axis is replaced by the effective aspect ratio, $\tilde{c}$, which is obtained from the values of $\beta$. The parameters $a=1, \lambda=2.5$ and $b=0.25$ are fixed. For seven different $c$, we plotted the values of $\alpha$ and $\beta$ as we increased $L$ from 0 to 100 , and the plotted circles indicate the values with $L=0,2, \ldots, 10$, with the same colours for the same value of $L$.

To obtain swimming trajectories, we need to compute the swimming velocity, which is parallel to the director vector. The detailed values could, however, depend on the modelling of the active part of the cell. If we consider a given rotational velocity for the flagellum in the body-fixed frame, the active force and torque, $F_{0}$ and $M_{0}$, should depend on the components of the linear and angular velocities parallel to the director vector (Lauga et al. 2006). In such a case, the equations (3.9)-(3.10) only provide implicit relations to determine the propulsive velocities. Nonetheless, as the director dynamics are decoupled from the active effects, the bacterial turning behaviour in a flow emerges purely from the geometry of the swimmer.

\section{Discussions and conclusions}

We have considered the dynamics of a general helicoidal object, a particle or swimmer with a $\pi / 2$-rotational symmetry around an axis referred to as helicoidal symmetry, in a linear background flow at low Reynolds number, and derived an equation for the director vector of the object, a generalized version of the Jeffery equation (1.1). This new equation includes two coefficients that reflect the shape of the object: the Bretherton constant $\beta$, and a new constant $\alpha$ derived from the chirality of the object.

The helicoidal symmetry description is reasonable for a rigid helix, and a squirmer with a helicoidal shape is also a helicoidal object. Moreover, considering the time-averaged behaviours of the self-propelling particles, it is also possible to model these swimmers as helicoidal objects under a moderate background flow condition. An example of a general helicoidal object is a bacterium-like particle or a swimmer (Marcos et al. 2012) that does not possess additional symmetries (figures $2 b$ and $3 b$ ). Since the propulsive effect of swimmers is decoupled from the director dynamics and from the drift velocity, the chiral Jeffery equation (1.1) therefore provides the general dynamics of a helicoidal particle and a swimmer. 


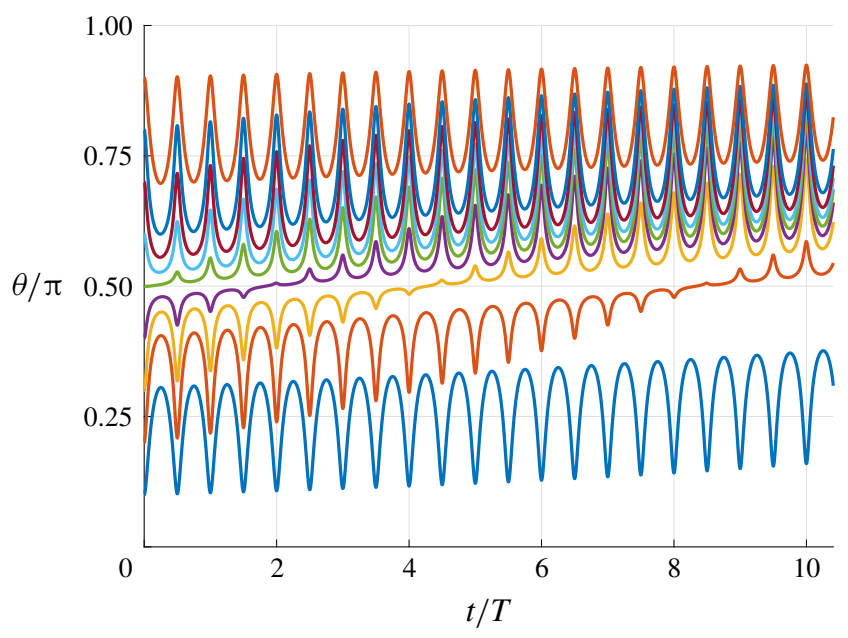

FIgURE 9. Time evolution of the polar angle $\theta$ with different initial values of $\theta_{\text {init }}=$ $0.1 \pi, 0.2 \pi, \ldots, 0.9 \pi$ against time normalized by the period of the Jeffery orbit $T$. The values of $\alpha$ and $\beta$ are $(\beta, \alpha)=(0.9,0.005)$, corresponding to values for a typical bacterial cell as obtained from the calculation in figure 8 .

Our new equation includes some previously known results on the angular dynamics of a chiral object. A helicoidal object with reflectional symmetry in a plane including an axis of helicoidal symmetry (figures $2 a, 3 a$ ) is a non-chiral object and follows the closed Jeffery orbits of any body of revolution (Bretherton 1962; Brenner 1964b). Further extensions for an object with discrete symmetry have been performed by Fries et al. (2017). A helical object with a fore-aft symmetry, such as a rod-like object with two connected helices of opposite chirality (Kramel et al. 2015), is included in the symmetry group of helicoidal objects with reflectional symmetry with respect to a plane perpendicular to the axis of helicoidal symmetry (figures $2 c, 3 c$ ), and we have proved in a general manner that such an object needs a constant $\alpha$ in the generalized Jeffery equations, though no drift velocity is generated. A rod-like helicoidal object considered in Chen \& Zhang (2011) belongs to the class of helicoidal objects with additional $\pi$-rotational symmetry around an axis perpendicular to the axis of helicoidal symmetry, as illustrated in figures $2(d), 3(d)$, and our generalized version of the Jeffery equations is reduced to the usual Jeffery equation as obtained by Chen \& Zhang (2011).

Comparing the results of the symmetry group of figures $2(c), 3(c)$ and figures $2(d)$, $3(d)$, it is found that the non-zero $\alpha$ results not from the overall chirality of the object but from the inhomogeneous distribution of the chiral effects along the axis of helicoidal symmetry. These results generalize the physical interpretation of the torque generation for the bacterial turning given by Marcos et al. (2012), where the chiralityinduced hydrodynamic force was generated only on the flagellar end. The overall chirality of the object contributes to the drift velocity but does not directly appear in $\alpha$, as shown in the symmetry class of figures $2(d)$ and $3(d)$.

Further analyses have been made for the case of a simple shear flow, and the director dynamics were reduced to two-dimensional ordinary differential equations concerning the two angle variables. We studied the structure of the equations, and the director dynamics of a helicoidal object with a positive $\alpha$ are found to be stable 
when the director vector is aligned toward the background vorticity vector, whereas the orientation anti-parallel to the vorticity vector is unstable for an elongated object with $\beta>0$ and $\alpha^{2}+\beta^{2}<1$. This condition is usually satisfied for a bacterial swimmer with a left-handed flagellum from resistive force theory calculations, which successfully express the bacterial turning dynamics in a shear flow (Marcos et al. 2012). This also emphasizes the usability of the simple equations with two constant parameters to express the dynamics of chiral self-propelling particles in a flow. The applications of these simple equations are also possible in studying the rheology of particle and swimmer suspensions. If $\alpha^{2}+\beta^{2}>1, \alpha \neq 0$, the object orientation is attracted to one of the two stable fixed points in the phase space, whereas nonlinear periodic orbits are obtained when $\alpha=0$. These non-trivial fixed points are possible by the nonlinearity of the director equation (1.1), which suggests complicated dynamics in a general background flow such as Kolmogorov flow, $\mathrm{ABC}$ flow and isotropic turbulence (Gustavsson \& Biferale 2016; Kramel et al. 2015; Clifton et al. 2018).

The sign of $\alpha$ determines the direction of the stable orientation, and this is given by the chirality of the object shape, as demonstrated in the general framework and shown by calculations for a bacterial swimmer with a helical flagellar filament. Observing the numerator of the expression for $\alpha$ in (3.14), the scalars of the resistance tensors related to the sign of $\alpha$ are all found to be proportional to $\sigma(\xi-1) C_{T}$ from the expressions in the appendix B. Here, $\sigma= \pm 1$ is the chirality of the helix and $C_{T}<0$, and the anisotropic ratio $\xi=C_{N} / C_{T}$ is typically in the range $1.5-2$ for a smooth flagellum. It is known that an additional hairy structure on the flagellum can reduce the value of $\xi$ to be less than unity, such as the mastigonemes of algal micro-organisms (Holwill \& Sleigh 1967; Brennen 1975; Kobayashi et al. 2009; Namdeo et al. 2011), suggesting possibilities for the design of the sign of $\alpha$ with additional manipulations for microparticles and microrobots.

Further extensions of the current study may be possible to a general object with less symmetry, such as an ellipsoid (Jeffery 1922; Hinch \& Leal 1979), which is known to exhibit chaotic director dynamics (Yarin, Gottlieb \& Roisman 1997). A general formulation was made by Junk \& Illner (2007), but further investigations for the relation to the scalars of the resistance tensors will be useful, as has been done for some discrete but relatively higher symmetries (Fries et al. 2017). Another important extension is to include gravity effects. Even a simple helix generates a complex trajectory under gravity (Kim \& Rae 1991; Palusa et al. 2018), and simple director equations will be very useful considering the dynamics in a flow.

In conclusion, we have derived the generalized Jeffery equations and Jeffery orbits for any helicoidal objects and swimmers. The expressions are very simple and thus useful for the studies of microparticles and biological fluid problems. This study also sheds light on the importance of the symmetry of an object for its dynamical patterns in a flow at low Reynolds number.

\section{Acknowledgements}

K.I. is supported by JSPS-KAKENHI for Young Researchers (18K13456) and JST, PRESTO grant number JPMJPR1921, Japan. The author acknowledges the anonymous referees for their comments, which helped me to improve the manuscript. This work was supported by the Research Institute for Mathematical Sciences, an International Joint Usage/Research Center located in Kyoto University.

\section{Declaration of interests}

The author reports no conflict of interest. 


\section{Appendix A. Hydrodynamic force, torque and resistance tensors}

In this appendix, we provide detailed expressions for the hydrodynamic resistance tensors. We start with the resistance tensors associated with the rigid motions $\boldsymbol{K}, \boldsymbol{C}$ and $\boldsymbol{Q}$. With the decomposition of the surface force $\hat{\boldsymbol{f}}=\boldsymbol{\Sigma} \cdot \hat{\boldsymbol{U}}+\boldsymbol{\Pi} \cdot \hat{\boldsymbol{\Omega}}$ as in the main text, we obtain the following expressions by employing the Lorentz reciprocal theorem (Yariv 2006; Ishimoto \& Yamada 2012):

$$
\boldsymbol{K}=\int_{S} \boldsymbol{\Sigma} \mathrm{d} S, \quad \boldsymbol{C}=\int_{S} \boldsymbol{r} \times \boldsymbol{\Sigma} \mathrm{d} S=\int_{S} \boldsymbol{\Pi}^{\mathrm{T}} \mathrm{d} S, \quad \boldsymbol{Q}=\int_{S} \boldsymbol{r} \times \boldsymbol{\Pi} \mathrm{d} S . \quad(\mathrm{A} 1 a-c)
$$

Application of the Lorentz reciprocal theorem leads to the second equality for $C$ and the symmetric properties of the tensors, $\boldsymbol{K}=\boldsymbol{K}^{\mathrm{T}}$ and $\boldsymbol{Q}=\boldsymbol{Q}^{\mathrm{T}}$ (Happel \& Brenner 1983). Introducing the antisymmetric tensor $\boldsymbol{X}$ such that $\boldsymbol{r} \times \boldsymbol{\Sigma}=\boldsymbol{X} \cdot \boldsymbol{\Sigma}$, we simply rewrite the expression for two of the resistance tensors as

$$
\boldsymbol{C}=\int_{S} \boldsymbol{x} \cdot \boldsymbol{\Sigma} \mathrm{d} S \text { and } \boldsymbol{Q}=\int_{S} \boldsymbol{x} \cdot \boldsymbol{\Sigma} \cdot \boldsymbol{X}^{\mathrm{T}} \mathrm{d} S
$$

with symmetric properties $\boldsymbol{\Sigma}=\boldsymbol{\Sigma}^{\mathrm{T}}$ (Happel \& Brenner 1983) and $\boldsymbol{\Pi}=\boldsymbol{\Sigma} \cdot \boldsymbol{X}^{\mathrm{T}}$.

The expressions for the additional forces and torques imparted by the background flow are also obtained by using the Lorentz reciprocal theorem (Pozrikidis 1992). The force induced by the background flow, $\boldsymbol{F}^{\infty}$, is

$$
\boldsymbol{F}^{\infty}=-\int_{S} \boldsymbol{u}^{\infty} \cdot \boldsymbol{\Sigma} \mathrm{d} S=-\boldsymbol{K} \cdot \boldsymbol{U}^{\infty}-\boldsymbol{C}^{\mathrm{T}} \cdot \boldsymbol{\Omega}^{\infty}-\int_{S} \boldsymbol{r} \cdot \boldsymbol{E}^{\infty} \cdot \boldsymbol{\Sigma} \mathrm{d} S,
$$

where we have used the linear expansion of the background flow $\boldsymbol{u}^{\infty}=\boldsymbol{U}^{\infty}+\boldsymbol{\Omega}^{\infty} \times$ $\boldsymbol{r}+\boldsymbol{r} \cdot \boldsymbol{E}^{\infty}$. The last term of (A 3) therefore corresponds to the shear-force triadic term in (2.4):

$$
\Gamma: E^{\infty}=-\int_{S} \boldsymbol{r} \cdot \boldsymbol{E}^{\infty} \cdot \boldsymbol{\Sigma} \mathrm{d} S=-\int_{S} \boldsymbol{\Sigma}^{\mathrm{T}} \boldsymbol{r}: \boldsymbol{E}^{\infty} .
$$

Following the expressions of Brenner (1964b) that satisfy identity (2.6), we define the shear-force tensor by

$$
\boldsymbol{\Gamma}=-\frac{1}{2} \int_{S}\left[\boldsymbol{\Sigma}^{\mathrm{T}} \boldsymbol{r}+\left(\boldsymbol{\Sigma}^{\mathrm{T}} \boldsymbol{r}\right)^{\mathrm{T}}\right] \mathrm{d} S=-\frac{1}{2} \int_{S}\left[\boldsymbol{\Sigma} \boldsymbol{r}+(\boldsymbol{\Sigma} \boldsymbol{r})^{\mathrm{T}}\right] \mathrm{d} S,
$$

noting that the transpose of a triad refers to the exchange of the second and third indices as in the main text. The shear-torque triad can also be derived from the expression of the flow-induced torque,

$$
\boldsymbol{M}^{\infty}=-\int_{S} \boldsymbol{u}^{\infty} \cdot \boldsymbol{\Pi} \mathrm{d} S
$$

and we have

$$
\boldsymbol{\Lambda}=-\frac{1}{2} \int_{S}\left[\boldsymbol{\Pi}^{\mathrm{T}} \boldsymbol{r}+\left(\boldsymbol{\Pi}^{\mathrm{T}} \boldsymbol{r}\right)^{\mathrm{T}}\right] \mathrm{d} S=-\frac{1}{2} \int_{S}\left[\boldsymbol{X} \cdot \boldsymbol{\Sigma} \boldsymbol{r}+(\boldsymbol{X} \cdot \boldsymbol{\Sigma} \boldsymbol{r})^{\mathrm{T}}\right] \mathrm{d} S .
$$




\section{Appendix B. Expression of the resistance tensors for a bacterial swimmer}

In this appendix, we provide explicit forms for the scalars in the resistance tensors for a model bacterium with a spherical cell body and a helical flagellum with $n$ cycles. As shown in figure 7 , we consider a spheroid of semiaxes $c, a, a$, the first of which corresponds to the axis of the helicoidal symmetry, $\hat{\boldsymbol{e}}_{1}$. The flagellum is simply modelled as a helix with flagellar radius $d$, helical radius $b$ and pitch $\lambda$. The following calculations are exact only when $n$ is an integer. However, the same expressions are obtained even for non-integer values of $n$ after taking the average over the phase of the flagellum, and this is reasonable for a bacterial swimmer whose flagellum is rapidly rotating around the $\hat{\boldsymbol{e}}_{1}$ axis compared with the time scale of the swimming and the rotation by the background shear.

Taking the origin of the body-fixed frame as the centre of the spheroid, we have the centreline of the helix in a parameterized form,

$$
\boldsymbol{r}=-\left(c+\frac{\lambda}{2 \pi} \tau\right) \hat{\boldsymbol{e}}_{1}+(b \cos \tau) \hat{\boldsymbol{e}}_{2}+(\sigma b \sin \tau) \hat{\boldsymbol{e}}_{3}, \quad(0 \leqslant \tau \leqslant 2 \pi n),
$$

where $\sigma \in\{+1,-1\}$ represents the chirality of the helix and $\sigma=1$ corresponds to a left-handed helix as in Lauga et al. (2006). The parameter $\tau$ is related to the arc length $s$ by $s=\left(1+\epsilon^{2}\right)^{1 / 2} \lambda \tau /(2 \pi)$ with $\epsilon=2 \pi b / \lambda$, and we introduce the effective length of the flagellum, $L=2 \pi n \lambda$. The tangent vector of the centreline is obtained as

$$
\boldsymbol{t}=\frac{\mathrm{d} \boldsymbol{r}}{\mathrm{d} s}=\cos \Psi \hat{\boldsymbol{e}}_{1}+\sin \Psi\left(-\sin \tau \hat{\boldsymbol{e}}_{2}+\sigma \cos \tau \hat{\boldsymbol{e}}_{3}\right),
$$

where $\Psi$ is the angle between the tangent vector and the $\hat{\boldsymbol{e}}_{1}$ axis, i.e., $\cos \Psi=$ $1 / \sqrt{1+\epsilon^{2}}$ and $\sin \Psi=\epsilon / \sqrt{1+\epsilon^{2}}$.

Within the resistive force theory, the hydrodynamic forces on a segment of the flagellum are linearly related to the local velocity $\boldsymbol{u}$ as

$$
\mathrm{d} \boldsymbol{F}(s)=\left[C_{T} \boldsymbol{t t}+C_{N}(\boldsymbol{I}-\boldsymbol{t t})\right] \cdot \boldsymbol{u}(s) \mathrm{d} s,
$$

using the tangential and normal drag coefficients, $C_{T}$ and $C_{N}$, both of which are negative constants along the flagellum. This expression (B 3) provides the translational surface force resistance tensor for a flagellum,

$$
\Sigma=C_{T} t \boldsymbol{t}+C_{N}(I-t \boldsymbol{t})
$$

By introducing the ratio of the coefficients from the resistive force theory $\xi=C_{N} / C_{T}$, from (B 2), we obtain the explicit form with respect to the body-fixed frame, $\Sigma_{i j} / C_{T}=$

$$
\left(\begin{array}{ccc}
\xi-(\xi-1) \cos ^{2} \Psi & -(\xi-1) \sin \Psi \cos \Psi \sin \tau & (\xi-1) \sigma \sin \Psi \cos \Psi \cos \tau \\
-(\xi-1) \sin \Psi \cos \Psi \sin \tau & \xi-(\xi-1) \sin ^{2} \Psi \sin ^{2} \tau & (\xi-1) \sigma \sin ^{2} \Psi \sin \tau \cos \tau \\
(\xi-1) \sigma \sin \Psi \cos \Psi \cos \tau & (\xi-1) \sigma \sin ^{2} \Psi \sin \tau \cos \tau & \xi-(\xi-1) \sin ^{2} \Psi \cos ^{2} \tau
\end{array}\right)
$$

The translational tensor $\boldsymbol{K}$ is obtained by integrating over the flagellum with additional terms from the spheroidal cell body, and the other scalars are also calculated by similar integrations. Introducing the wavenumber of the helix $k=2 \pi / \lambda$, after some lengthy but straightforward calculations of (A 1), we have the values for the scalars for the translational tensor,

$$
K_{1}=\frac{C_{T}}{\sqrt{1+\epsilon^{2}}}\left(1+\xi \epsilon^{2}\right) L+K_{1}^{H}
$$




$$
K_{2}=\frac{C_{T}}{\sqrt{1+\epsilon^{2}}}\left[\xi+\left(\frac{1}{2}+\frac{\xi}{2}\right)\right] L+K_{2}^{H},
$$

for the rotational tensor,

$$
\begin{gathered}
Q_{1}=\frac{-C_{T}}{\sqrt{1+\epsilon^{2}}}\left(\xi+\frac{\epsilon^{2}}{4}\right) L b^{2}+Q_{1}^{H}, \\
Q_{2}=\frac{C_{T}}{\sqrt{1+\epsilon^{2}}}\left[\xi+\left(\frac{1}{2}+\frac{\xi}{2}\right)\right]\left(c^{2}+c L+\frac{L^{2}}{3}\right) L \\
+\frac{C_{T}}{\sqrt{1+\epsilon^{2}}} \frac{1}{2}\left(1+\xi \epsilon^{2}\right) L b^{2}+Q_{2}^{H},
\end{gathered}
$$

and for the coupling tensor,

$$
\begin{gathered}
C_{1}=\frac{\sigma C_{T}}{\sqrt{1+\epsilon^{2}}}(\xi-1) L b^{2} k, \\
C_{2}=\frac{-\sigma C_{T}}{\sqrt{1+\epsilon^{2}}} \frac{1}{2}(\xi-1) L b^{2} k, \\
C_{23}=\frac{C_{T}}{\sqrt{1+\epsilon^{2}}}\left[\xi+\left(\frac{1}{2}+\frac{\xi}{2}\right)\right]\left(c+\frac{L}{2}\right) L,
\end{gathered}
$$

and similar calculations will lead to the expressions of the scalars for the shear-force tensor,

$$
\begin{gathered}
\Gamma_{1}=\frac{C_{T}}{\sqrt{1+\epsilon^{2}}}\left(1+\xi \epsilon^{2}\right)\left(c+\frac{L}{2}\right) L, \\
\Gamma_{2}=\frac{C_{T}}{\sqrt{1+\epsilon^{2}}}\left[\xi+\left(\frac{1}{2}+\frac{\xi}{2}\right)\right]\left(c+\frac{L}{2}\right) L, \\
\Gamma_{3}=\frac{\sigma C_{T}}{\sqrt{1+\epsilon^{2}}} \frac{1}{2}(\xi-1) L b^{2} k,
\end{gathered}
$$

from (A 5) and (3.1), and those of the shear-torque tensor,

$$
\begin{gathered}
\Lambda_{1}=\frac{\sigma C_{T}}{\sqrt{1+\epsilon^{2}}}(\xi-1)\left(c+\frac{L}{2}\right) L b^{2} k, \\
\Lambda_{2}=\frac{-\sigma C_{T}}{\sqrt{1+\epsilon^{2}}}(\xi-1)\left(c+\frac{L}{2}\right) L b^{2} k, \\
\Lambda_{3}=\frac{C_{T}}{\sqrt{1+\epsilon^{2}}}\left[\xi+\left(\frac{1}{2}+\frac{\xi}{2}\right)\right]\left(c^{2}+c L+\frac{L^{2}}{3}\right) L \\
-\frac{C_{T}}{\sqrt{1+\epsilon^{2}}} \frac{1}{2}\left(1+\xi \epsilon^{2}\right) L b^{2}+\Lambda_{3}^{H},
\end{gathered}
$$

from (A 7) and (3.2), where the terms from the spheroidal cell-body, $K_{1}^{H}, K_{2}^{H}, Q_{1}^{H}, Q_{2}^{H}$ and $\Lambda_{3}^{H}$, are explicitly shown in Kim \& Karrila (2005). These expressions include the formula from Lauga et al. (2006) in the absence of a wall if we set $\xi=2$. When the flagellum is removed (or if simply $L=0$ ), the coefficients for the director equation (3.14) are reduced to the well known results of that of a spheroid, $\alpha=0$ 
and $\beta=\Lambda_{3}^{H} / K_{2}^{H}=\left[1-(a / c)^{2}\right] /\left[1+(a / c)^{2}\right]$. At the long flagellum limit, we have $\alpha \rightarrow 0$ and $\beta \rightarrow 1$.

\section{REFERENCES}

Aristov, M., EICHhorn, R. \& Bechinger, C. 2013 Separation of chiral colloidal particles in a helical flow field. Soft Matt. 9, 2525-2530.

BATCHELOR, G. K. 1970 Slender-body theory for particles of arbitrary cross-section in Stokes flow. J. Fluid Mech. 44, 419-449.

Bearon, R. N., Hazel, A. L. \& Thron, G. J. 2011 The spatial distribution of gyrotactic swimming micro-organisms in laminar flow fields. J. Fluid Mech. 680, 602-635.

Brennen, C. 1975 Locomotion of flagellates with mastigonemes. J. Mechanochem. Cell Motil. 3, 207-221.

BREnNer, H. 1964a The Stokes resistance of an arbitrary particle - II an extension. Chem. Engng Sci. 19, 599-629.

BREnNer, H. $1964 b$ The Stokes resistance of an arbitrary particle - III shear fields. Chem. Engng Sci. 19, 631-651.

BRetherton, F. B. 1962 The motion of rigid particles in a shear flow at low Reynolds number. J. Fluid Mech. 14, 284-304.

Chen, P. \& Zhang, Q. 2011 Dynamical solutions for migration of chiral DNA-type objects in shear flows. Phys. Rev. E 84, 056309.

Chwang, A. T. \& WU, T. Y. 1971 A note on the helical movement of micro-organisms. Proc. $R$. Soc. Lond. B 178, 327-346.

Clifton, W., Bearon, R. N. \& Bees, M. A. 2018 Enhanced sedimentation of elongated plankton in simple flows. IMA J. Appl. Maths 83, 743-766.

CRowdy, D. 2016 Flipping and scooping of curved 2D rigid fibers in simple shear: the Jeffery equations. Phys. Fluids 28, 053105.

Doi, M. \& Makino, M. 2005 Motion of micro-particles of complex shape. Prog. Polym. Sci. 30, 876-884.

EICHHORn, R. 2010 Microfluidic sorting of stereoisomers. Phys. Rev. Lett. 105, 034502.

Fries, J., EINARSSON, J. \& MEHLIG, B. 2017 Angular dynamics of small crystals in viscous flow. Phys. Rev. Fluids 2, 014302.

GRAY, J. \& HANCOCK, G. J. 1955 The propulsion of sea-urchin spermatozoa. J. Expl Biol. 32, $802-814$.

Gustavsson, K. \& Biferale, L. 2016 Preferential sampling of helicity by isotropic helicoids. Phys. Rev. Fluids 1, 054201.

Happel, J. \& Brenner, H. 1983 Low Reynolds Number Hydrodynamics with Special Applications to Particular Media. Martinus Nijhoff Publishers.

Hermans, T. M., Bishop, K. J. M., Stewart, P. S., Davis, S. H. \& Grzybowski, B. A. 2015 Vortex flows impart chirality-specific lift forces. Nat. Commun. 6, 5640.

Hill, J., Kalkanci, O., MCMurry, J. L. \& Koser, H. 2007 Hydrodynamic surface interactions enable Escherichia Coli to seek efficient routes to swim upstream. Phys. Rev. Lett. 98, 068101.

HINCH, E. J. \& LEAL, L. G. 1972 The effect of Brownian motion on the rheological properties of a suspension of non-spherical particles. J. Fluid Mech. 62, 683-712.

HINCH, E. J. \& LEAL, L. G. 1979 Rotation of small non-axisymmetric particles in a simple shear flow. J. Fluid Mech. 92, 591-608.

Holwill, M. E. J. \& Sleigh, M. A. 1967 Propulsion by hispid flagella. J. Expl Biol. 47, 267-276.

Isнiмото, K. 2017 Guidance of microswimmers by wall and flow: thigmotaxis and rheotaxis of unsteady squirmers in two and three dimensions. Phys. Rev. E 96, 043101.

Isнiмото, K. 2019 Bacterial spinning top. J. Fluid Mech. 880, 620-652.

Ishimoto, K. \& GAfFney, E. A. 2013 Squirmer dynamics near a boundary. Phys. Rev. E 88, 062702 . 
Ishimoto, K. \& GAFFney, E. A. 2015 Fluid flow and sperm guidance: a simulation study of hydrodynamic sperm rheotaxis. J. R. Soc. Interface 12, 20150172.

Ishimoto, K. \& LAUGA, E. 2019 The N-flagella problem: eastohydrodynamic motility transition of multi-flagellated bacteria. Proc. R. Soc. Lond. A 475, 20180690.

Ishimoto, K. \& YAmadA, M. 2012 A coordinate-based proof of the Scallop theorem. SIAM J. Appl. Maths 72, 1686-1694.

Jeffery, G. B. 1922 The motion of ellipsoidal particle immersed in a viscous fluid. Proc. R. Soc. Lond. A 102, 161-179.

Junk, M. \& Illner, R. 2007 A new derivation of Jeffery's equation. J. Math. Fluid Mech. 9, $455-488$.

Kantsler, V., Dunkel, J., Blayney, M. \& Goldstein, R. E. 2014 Rheotaxis facilitates upstream navigation of mammalian sperm cell. eLife 3, e02403.

Kaya, T. \& Kose, H. 2012 Direct upstream motility in Escherichia Coli. Biophys. J. 102, 1514-1523.

Kelvin, Lord 1871 Hydrokinetic solutions and observations. Phil. Mag. 42, 362-377.

Kim, S. \& Karrila, S. J. 2005 Microhydrodynamics: Principles and Selected Applications. Dover Publications.

KIM, Y. J. \& RAE, W. J. 1991 Separation of screw-sensed particles in a homogeneous shear field. Intl J. Multiphase Flow 17, 714-744.

KIRChHOFF, G. 1869 Uber die Benegung eines Rotationskörpers in earner Flüssigkeit. J. Reine Angew. Math. 71, 237-262.

Kobayashi, S., Watanabe, R., Oiwa, T. \& Morikawa, H. 2009 Computational study of micropropulsion mechanism in water modeled on flagellum with projecting mastigonemes. J. Biomech. Sci. Engng 4, 11-22.

Kramel, S., Voth, G. A., Tympel, S. \& Tympe, S. 2015 Preferential rotation of chiral dipoles in isotropic turbulence. Phys. Rev. Lett. 117, 154501.

LAmB, H. 1932 Hydrodynamics, 6th edn. Dover Publications.

LAMOR, J. 1884 On hydro-kinetic symmetry. Q. J. Pure Appl. Maths 20, 261-265.

Lauga, E., Diluzio, W. R., Whiteside, G. M. \& Stone, H. A. 2006 Swimming in circles: motion of bacteria near solid boundaries. Biophys. J. 90, 400-412.

Makino, M. \& DoI, M. 2005 Migration of twisted ribbon-like particles in simple shear flow. Phys. Fluids 17, 103605.

Marcos, Fu, H. C., Powers, T. R. \& Stocker, R. 2009 Separation of microscale chiral objects by shear flow. Phys. Rev. Lett. 102, 158103.

Marcos, Fu, H. C., Powers, T. R. \& Stocker, R. 2012 Bacterial rheotaxis. Proc. Natl Acad. Sci. USA 109, 4780-4785.

MAXEY, M. R. \& Riley, J. J. 1983 Equation of motion for a small rigid sphere in a nonuniform flow. Phys. Fluids 26, 883-889.

Mueller, S., Llwwelln, E. W. \& Mader, H. M. 2010 The rheology of suspensions of solid particles. Proc. R. Soc. Lond. A 466, 1201-1228.

Namdeo, S., Khaderi, S. N., Den Toonder, J. M. J. \& ONCK, P. R. 2011 Swimming direction reversal of flagella through ciliary motion of mastigonemes. Biomicrofluidics 5, 034108.

PAK, O. S. \& LAUGA, E. 2014 Generalized squirming motion of a sphere. J. Engng Maths 88, $1-28$.

Palusa, M., de Graaf, J., Brown, A. \& Morozov, A. 2018 Sedimentation of a rigid helix in viscous media. Phys. Rev. Fluids 3, 124301.

Pedley, T. J., BRumley, D. R. \& Goldstein, R. E. 2016 Squirmers with swirl: a model for volvox swimming. J. Fluid Mech. 798, 165-186.

Pedley, T. J. \& Kessler, J. O. 1992 Hydrodynamic phenomena in suspensions of swimming microorganisms. Annu. Rev. Fluid Mech. 24, 313-358.

Petrie, C. J. S. 1999 The rheology of fibre suspensions. J. Non-Newtonian Fluid Mech. 87, 369-402.

PozRIKIDIs, C. 1992 Boundary Integral and Singularity Methods. Cambridge University Press.

Purcell, E. M. 1977 Life at low Reynolds number. Am. J. Phys. 45, 3-11.

Riley, E. E., DAS, D. \& LAUGA, E. 2018 Swimming of peritrichous bacteria is enabled by an elastohydrodynamic instability. Sci. Rep. 8, 10728. 
Ro, S., YI, J. \& Kim, Y. W. 2016 Chiral separation by flows: the role of flow symmetry and dimensionality. Sci. Rep. 6, 35144.

Rodenborn, B., Chen, C.-H., Swinney, H. L., Liu, B. \& Zhang, P. 2013 Propulsion of microorganisms by a helical flagellum. Proc. Natl Acad. Sci. USA 110, E338-E347.

Spagnolie, S. E. \& LAUGa, E. 2012 Hydrodynamics of self-propulsion near a boundary: prediction and accuracy of far-field approximations. J. Fluid Mech. 700, 105-147.

Stokes, G. G. 1851 On the effect of internal friction of fluids on the motion of pendulums. Trans. Camb. Phil. Soc. 9, 8-106.

Tillett, J. P. K. 1970 Axial and transverse Stokes flow past slender axisymmetric bodies. J. Fluid Mech. 44, 401-417.

Uspal, W. E., Popescu, M. N., Dietrich, S. \& Tasinkevych, M. 2015 Rheotaxis of spherical active particles near a planar wall. Soft Matt. 11, 6613-6632.

Voth, G. A. \& Soldati, A. 2017 Anisotropic particles in turbulence. Annu. Rev. Fluid Mech. 49, 249-276.

Walker, B. J., Ishimoto, K., Wheeler, R. J. \& Gaffney, E. A. 2018 Response of monoflagellate pullers to a shearing flow: a simulation study of microswimmer guidance. Phys. Rev. E 98, 063111.

Yarin, A. L., Gottlieb, O. \& Roisman, I. V. 1997 Chaotic rotation of triaxial ellipsoids in simple shear. J. Fluid Mech. 340, 83-100.

YARIV, E. 2006 Self-propulsion in a viscous fluid: arbitrary surface deformations. J. Fluid Mech. 550, 139-148.

Zöttl, A.\& STARK, H. 2013 Periodic and quasiperiodic motion of an elongated microswimmer in Poiseuille flow. Eur. Phys. J. E 36, 4. 